\title{
Genome-wide identification of cyclophilin genes in Gossypium hirsutum and functional characterization of a CYP with antifungal activity against Verticillium dahliae
}

Jun Yang, Guoning Wang, Huifeng Ke, Yan Zhang, Lianlian Ji, Lizhi Huang, Chunying Zhang, Xingfen Wang* and Zhiying $\mathrm{Ma}^{*}$

\begin{abstract}
Background: Cyclophilins (CYPs), belonging to the peptidyl prolyl cis/trans isomerase (PPlase) superfamily, play important roles during plant responses to biotic and abiotic stresses.

Results: Here, a total of 79 CYPs were identified in the genome of Gossypium hirsutum. Of which, 65 GhCYPs only contained one cyclophilin type PPlase domain, others 14 GhCYPs contain additional domains. A number of cis-acting elements related to phytohormone signaling were predicated in the upstream of GhCYPs ORF. The expression analysis revealed that GhCYPs were induced in response to cold, hot, salt, PEG and Verticillium dahliae infection. In addition, the functional importance of GhCYP-3 in Verticillium wilt resistance was also presented in this study. GhCYP-3 showed both cytoplasmic and nuclear localization. Overexpression of GhCYP-3 in Arabidopsis significantly improved Verticillium wilt resistance of the plants. Recombinant GhCYP-3 displayed PPlase activity and evident inhibitory effects on $V$. dahliae in vitro. Moreover, the extracts from GhCYP-3 transgenic Arabidopsis displayed significantly inhibit activity to conidia germinating and hyphal growth of $V$. dahliae.

Conclusions: Our study identified the family members of cotton CYP genes using bioinformatics tools. Differential expression patterns of GhCYPs under various abiotic stress and $V$. dahliae infection conditions provide a comprehensive understanding of the biological functions of candidate genes. Moreover, GhCYP-3 involved in the resistance of cotton to $V$. dahliae infection presumably through antifungal activity.
\end{abstract}

Keywords: Cyclophilin, Cotton, Resistance, Verticillium dahliae, Antifungal activity

\section{Background}

The allotetraploid Gossypium hirsutum L., the most important fiber crop, is cultivated worldwide because of its high yield [1]. Environmental stresses, such as cold, drought, heat, salinity, various pests and pathogens, threaten cotton growth, yield and fiber quality. For example, an area of about 300 million hectares of cotton is subject to Verticillium wilt and the economic loss is more than RMB 12 billion every year in China. Verticillium wilt,

\footnotetext{
* Correspondence: cotton@hebau.edu.cn; mzhy@hebau.edu.cn North China Key Laboratory for Crop Germplasm Resources of Education Ministry, Hebei Agricultural University, Baoding 071001, China
}

the most serious disease to influence cotton production in China, caused by soil-borne fungus Verticillium dahliae. At present, few resistant varieties have been cultivated by traditional cross-breeding in G. hirsutum, which contributes $95 \%$ of the total cotton yield in the world [2]. Genetic engineering using plant resistance genes is becoming an alternative to improve cotton resistance to $V$. dahliae.

Cyclophilins (CYPs), possessing peptidyl prolyl cis/trans isomerase (PPIase) activity, are classified in the immunophilin family of proteins [3]. CYPs play important roles in various biological processes, including transcription regulation [4], protein folding [5], signal transduction [6] and

(c) The Author(s). 2019 Open Access This article is distributed under the terms of the Creative Commons Attribution 4.0 International License (http://creativecommons.org/licenses/by/4.0/), which permits unrestricted use, distribution, and 
ROS (reactive oxygen species) regulation [7]. With the availability of whole genome sequencing, the identification and characterization of plant CYPs are carried out mainly in Arabidopsis thaliana (29 AtCYPs) [8], Oryza sativa (27 OsCYPs) [9] and Glycine $\max$ (62 GmCYPs) [10]. The majority of studies reveal the involvement of plant CYPs mostly in different types of abiotic stress. For example, Arabidopsis CyPs showed evidence of response to wounding [11]. Rice OsCYP19-4 showed over 10-fold upregulation in response to cold. Overexpressing of OsCYP19-4 could enhance rice plants cold-resistance with significantly increased tiller and spike numbers, and consequently enhanced grain weight [12]. Transgenic plants overexpressing OsCYP21-4 exhibited increased tolerance to salinity and hydrogen peroxide treatment [13]. Ectopic expression of pigeon pea (Cajanus cajan L.) CYP, CcCYP, in Arabidopsis exhibited high-level tolerance against drought, salinity and extreme temperatures [14]. Against biotic stress, especially against pathogen infection, only several plant CYPs have been studied in plant-pathogen system. Pepper cyclophilin (CACYP1) gene expression increased in response to Xanthomonas campestris pv. vesicatoria and Colletotrichum gloeosporioides [15]. Fungal infection with Fusariumsolani f. speumartii increased the level of Solanum tuberosusm CyP gene StCyP mRNA in tubers [16]. The expression of $V$. vinifera $V v i C y P$ was highly induced by Plasmopara viticola [17]. In cotton, a cyclophilin-like gene GhCyp1 was cloned from G. hirsutum. Overexpression of GhCyp1 in transgenic tobacco plants conferred higher tolerance to salt stress and Pseudomonas syringae pv. tabaci infection compared with control plants [18].

In 2015, the genome of G. hirsutum L. acc. Texas Marker-1 (TM-1) was sequenced, more than 70, 000 protein-coding genes were predicted (NAU version 1.1) $[19,20]$. Recently, an improved de novo-assembled genome for G. hirsutum L. acc. TM-1 were generated (NAU version 2.1) [21]. The genome-sequencing project facilitates the survey of all CYP genes in cotton. In the present study, the CYP gene family members in G. hirsutum and their expression patterns under various abiotic stresses and on $V$. dahliae infection were systematically investigated. Furthermore, the function of GhCYP-3 was analyzed to reveal its role in cotton resistance to $V$. dahliae infection. Our study will enlighten the novel insights into the function of CYP genes in plant against multivariate stress responses in the future and provide more candidate genes for resistance breeding in cotton.

\section{Results}

Up to 79 CYPs were identified in the genome of $G$. hirsutum TM-1

A local BLASTP search was performed with the Arabidopsis CYP proteins as query, which resulted in 79 CYP candidates from G. hirsutum NAU version 1.1, 74 CYPs from G. hirsutum JGI version 1.0 and 78 CYPs from G. hirsutum NAU version 2.1 (Table 1). These candidates were submitted to Pfam to confirm the existence of cyclophilin type PPIase domain (CLD, PF00160) and named GhCYP-1 to GhCYP-79. The characteristics of the individual CYP, including CDS length, protein length, molecular weight, and isoelectric point (pI) were presented in Table 1. The protein length varied from 69 amino acid (aa) residues (GhCYP-70) to 801 aa (GhCYP-55). The molecular weight ranged from $7.5 \mathrm{kDa}$ (GhCYP-70) to 90.5 $\mathrm{kDa}$ (GhCYP-55), and the $\mathrm{pI}$ values ranged from 4.6 to 12.0. Most of the GhCYPs were expected to be in the cytoplasm. Also, some GhCYPs exhibited chloroplast, mitochondrial, nuclear and extracellular localization. Of the 79 GhCYPs, 65 only contained one CLD domain, but the remaining 14 GhCYPs contain additional domains, including tetratricopeptide-like repeats (TPR, PF00515, PF07719, PF13181, PF13414), Zinc finger (zf-CCHC, PF00098), RNA recognition motif (RRM, PF00076) and WD40 (PF00400) (Fig. 1).

\section{Cis-elements potentially related to hormonal signal for GhCYPs}

Here we surveyed the presence of cis-elements potentially related to the hormonal signal, in the $-2 \mathrm{~kb} 5^{\prime}$ flanking region upstream to the start codon of these GhCYPs. In total six types of hormones related cis-elements in the promoters were predicted (Fig. 2). Of these GhCYPs, 66 GhCYPs had ethylene (ET) responsive element (ERE), 35 GhCYPs contained salicylic acid (SA) responsive element (TCA-element), 47 GhCYPs harbored abscisic acid (ABA) responsive element (ABRE), 38 GhCYPs possessed gibberellin (GA) responsive element (P-box; TATC-box), 47 GhCYPs contained methyl jasmonate (MeJA) responsive element (CGTCA-motif), and 26 possessed auxin responsive element (TGA-box; AuxRR-core). In total, 217 cis-elements related to ET, 150 cis-elements related to MeJA, 132 cis-elements related to ABA, 57 cis-elements related to GA, 43 cis-elements related to SA and 34 cis-elements related to auxin were identified in all GhCYPs (Fig. 2). The enrichment of hormone-responsive cis-elements in the upstream of these GhCYPs suggests that they are likely to be involved in plant responses to various hormone signal pathways.

\section{Expression patterns of GhCYPs under various abiotic stresses}

Expression profiles of GhCYPs were examined in roots of cotton plants under four different abiotic stress conditions using high-throughput RNA-seq data (Additional file 2: Table S2). The transcripts with low Fragments Per Kilobase of exon per Million fragments mapped (FPKM) were probably false assembly. Therefore, in this study GhCYPs with FPKM $>10$ and present in at least two 
Table 1 The list of the putative CYP genes identified in G. hirsutum

\begin{tabular}{|c|c|c|c|c|c|c|c|c|}
\hline $\begin{array}{l}\text { Gene } \\
\text { Name }\end{array}$ & Gene ID (NAU1) & $\begin{array}{l}\text { CDS } \\
\text { (bp) }\end{array}$ & $\begin{array}{l}\text { Protein } \\
\text { (aa) }\end{array}$ & $\begin{array}{l}\mathrm{MW} \\
(\mathrm{kDa})\end{array}$ & $\mathrm{pl}$ & $\begin{array}{l}\text { Subcellular } \\
\text { location }\end{array}$ & Gene ID (JGI/NAU2) & $\begin{array}{l}\text { Identity (\%) (JGI/ } \\
\text { NAU2) }\end{array}$ \\
\hline GhCYP-1 & Gh_A01G0027 & 675 & 224 & 24.5 & 7.3 & Cyto & $\begin{array}{l}\text { Gohir.A01G003000/ } \\
\text { GH_A01G0029 }\end{array}$ & $100 / 100$ \\
\hline GhCYP-2 & Gh_A01G0031 & 516 & 171 & 18.2 & 7.9 & Cyto & $\begin{array}{l}\text { Gohir.A01G003400/ } \\
\text { GH_A01G0034 }\end{array}$ & 99/99 \\
\hline GhCYP-3 & Gh_A01G1361 & 522 & 173 & 18.2 & 8.5 & Cyto & $\begin{array}{l}\text { Gohir.A01G164200/ } \\
\text { GH_A01G1763 }\end{array}$ & $100 / 100$ \\
\hline GhCYP-4 & Gh_A01G1747 & 495 & 164 & 18.0 & 8.2 & Cyto/Mito/Chlo & $\begin{array}{l}\text { Gohir.A01G204600/ } \\
\text { GH_A01G2217 }\end{array}$ & $100 / 100$ \\
\hline GhCYP-5 & Gh_A02G0528 & 708 & 235 & 26.5 & 9.8 & Mito & $\begin{array}{l}\text { Gohir.A02G054200/ } \\
\text { GH_A02G0571 }\end{array}$ & $100 / 100$ \\
\hline GhCYP-6 & Gh_A02G1526 & 1116 & 371 & 41.9 & 6.6 & Cyto & No found/GH_A02G1850 & $-/ 100$ \\
\hline GhCYP-7 & Gh_A03G0499 & 966 & 321 & 35.0 & 8.7 & Chlo & $\begin{array}{l}\text { Gohir.A03G057400/ } \\
\text { GH_A03G0699 }\end{array}$ & $100 / 100$ \\
\hline GhCYP-8 & Gh_A03G0865 & 648 & 216 & 24.5 & 6.1 & Chlo & $\begin{array}{l}\text { Gohir.A03G099700/ } \\
\text { GH_A03G1148 }\end{array}$ & $100 / 100$ \\
\hline GhCYP-9 & Gh_A03G1688 & 1221 & 406 & 45.9 & 8.8 & Cyto & $\begin{array}{l}\text { Gohir.A03G191300/ } \\
\text { GH_A03G2153 }\end{array}$ & $100 / 100$ \\
\hline GhCYP-10 & Gh_A04G1046 & 522 & 173 & 18.5 & 10.1 & Cyto & No found/GH_A04G1474 & $-/ 100$ \\
\hline GhCYP-11 & Gh_A04G1047 & 522 & 173 & 18.3 & 8.2 & Cyto & No found/GH_A04G1475 & $-/ 100$ \\
\hline GhCYP-12 & Gh_A05G0642 & 1026 & 341 & 37.6 & 8.6 & Cyto & $\begin{array}{l}\text { Gohir.A05G078500/ } \\
\text { GH_A05G0800 }\end{array}$ & $74 / 73$ \\
\hline GhCYP-13 & Gh_A05G3461 & 567 & 188 & 20.4 & 8.2 & Cyto & No found/GH_A05G4196 & $-/ 100$ \\
\hline GhCYP-14 & Gh_A05G4019 & 1836 & 611 & 70.5 & 6.2 & $\mathrm{NuCl}$ & $\begin{array}{l}\text { Gohir.A05G003300/ } \\
\text { GH_A05G0027 }\end{array}$ & $100 / 100$ \\
\hline GhCYP-15 & Gh_A06G0418 & 870 & 289 & 31.9 & 6.9 & Extra & $\begin{array}{l}\text { Gohir.A06G049800/ } \\
\text { GH_A06G0538 }\end{array}$ & $100 / 100$ \\
\hline GhCYP-16 & Gh_A06G0767 & 1494 & 497 & 56.1 & 8.7 & Nucl & $\begin{array}{l}\text { Gohir.A06G085500/ } \\
\text { GH_A06G0934 }\end{array}$ & $100 / 100$ \\
\hline GhCYP-17 & Gh_A07G0324 & 2373 & 790 & 89.3 & 12.0 & $\mathrm{Nucl}$ & $\begin{array}{l}\text { Gohir.A07G038000/ } \\
\text { GH_A07G0439 }\end{array}$ & $100 / 99$ \\
\hline GhCYP-18 & Gh_A07G0325 & 2388 & 795 & 89.9 & 11.6 & Nucl & $\begin{array}{l}\text { Gohir.A07G038100/ } \\
\text { GH_A07G0440 }\end{array}$ & $93 / 93$ \\
\hline GhCYP-19 & Gh_A07G0986 & 1866 & 621 & 70.0 & 7.0 & Cyto & $\begin{array}{l}\text { Gohir.A07G108500/ } \\
\text { GH_A07G1 } 190\end{array}$ & $100 / 100$ \\
\hline GhCYP-20 & Gh_A07G2012 & 1359 & 452 & 50.0 & 4.9 & Chlo & $\begin{array}{l}\text { Gohir.A07G219400/ } \\
\text { GH_A07G2484 }\end{array}$ & $100 / 99$ \\
\hline GhCYP-21 & Gh_A08G0354 & 711 & 236 & 26.9 & 9.2 & Mito & $\begin{array}{l}\text { Gohir.A08G040200/ } \\
\text { GH_A08G0443 }\end{array}$ & $100 / 100$ \\
\hline GhCYP-22 & Gh_A08G1077 & 855 & 284 & 31.0 & 8.2 & Extra/PM/Chlo & $\begin{array}{l}\text { Gohir.A08G122000/ } \\
\text { GH_A08G1462 }\end{array}$ & $88 / 97$ \\
\hline GhCYP-23 & Gh_A08G1194 & 483 & 160 & 17.4 & 8.5 & Cyto & $\begin{array}{l}\text { Gohir.A08G133100/ } \\
\text { GH_A08G1597 }\end{array}$ & $100 / 100$ \\
\hline GhCYP-24 & Gh_A08G1470 & 1032 & 343 & 38.0 & 5.4 & Extra & $\begin{array}{l}\text { Gohir.A08G162600/ } \\
\text { GH_A08G1886 }\end{array}$ & $100 / 100$ \\
\hline GhCYP-25 & Gh_A08G1670 & 1986 & 661 & 72.8 & 10.7 & Nucl & $\begin{array}{l}\text { Gohir.A08G187500/ } \\
\text { GH_A08G2136 }\end{array}$ & $94 / 94$ \\
\hline GhCYP-26 & Gh_A09G0254 & 1308 & 435 & 47.9 & 4.6 & Cyto & $\begin{array}{l}\text { Gohir.A09G026100/ } \\
\text { GH_A09G0300 }\end{array}$ & $100 / 100$ \\
\hline GhCYP-27 & Gh_A09G0853 & 624 & 207 & 22.2 & 9.4 & Cyto/Mito & $\begin{array}{l}\text { Gohir.A09G090200/ } \\
\text { GH_A09G1082 }\end{array}$ & $96 / 96$ \\
\hline GhCYP-28 & Gh_A09G1765 & 1032 & 343 & 37.9 & 6.0 & Extra & $\begin{array}{l}\text { Gohir.A09G197200/ } \\
\text { GH_A09G2134 }\end{array}$ & $100 / 100$ \\
\hline
\end{tabular}


Table 1 The list of the putative CYP genes identified in G. hirsutum (Continued)

\begin{tabular}{|c|c|c|c|c|c|c|c|c|}
\hline $\begin{array}{l}\text { Gene } \\
\text { Name }\end{array}$ & Gene ID (NAU1) & $\begin{array}{l}\text { CDS } \\
(\mathrm{bp})\end{array}$ & $\begin{array}{l}\text { Protein } \\
\text { (aa) }\end{array}$ & $\begin{array}{l}\mathrm{MW} \\
(\mathrm{kDa})\end{array}$ & $\mathrm{pl}$ & $\begin{array}{l}\text { Subcellular } \\
\text { location }\end{array}$ & Gene ID (JGI/NAU2) & $\begin{array}{l}\text { Identity (\%) (JGI/ } \\
\text { NAU2) }\end{array}$ \\
\hline GhCYP-29 & Gh_A10G0832 & 1089 & 362 & 40.3 & 6.7 & Cyto & $\begin{array}{l}\text { Gohir.A10G092800/ } \\
\text { GH_A10G0930 }\end{array}$ & 97/96 \\
\hline GhCYP-30 & Gh_A10G1682 & 576 & 191 & 20.7 & 8.2 & Cyto & $\begin{array}{l}\text { Gohir.A10G187900/ } \\
\text { GH_A10G2053 }\end{array}$ & $100 / 100$ \\
\hline GhCYP-31 & Gh_A10G1687 & 609 & 202 & 22.1 & 8.6 & Cyto & $\begin{array}{l}\text { Gohir.A10G188300/ } \\
\text { GH_A10G2060 }\end{array}$ & $90 / 89$ \\
\hline GhCYP-32 & Gh_A10G2121 & 666 & 221 & 23.9 & 9.4 & Cyto & $\begin{array}{l}\text { Gohir.A10G236300/ } \\
\text { GH_A10G2615 }\end{array}$ & $100 / 100$ \\
\hline GhCYP-33 & Gh_A11G0678 & 996 & 331 & 37.2 & 5.3 & Extra & $\begin{array}{l}\text { Gohir.A11G074800/ } \\
\text { GH_A11G0773 }\end{array}$ & $82 / 82$ \\
\hline GhCYP-34 & Gh_A11G0987 & 705 & 234 & 26.8 & 8.9 & Mito/Nucl & $\begin{array}{l}\text { Gohir.A11G108500/ } \\
\text { GH_A11G1131 }\end{array}$ & $100 / 100$ \\
\hline GhCYP-35 & Gh_A12G0709 & 1791 & 596 & 65.3 & 8.2 & Mito/Nucl & $\begin{array}{l}\text { Gohir.A12G077300/ } \\
\text { GH_A12G0891 }\end{array}$ & $100 / 100$ \\
\hline GhCYP-36 & Gh_A12G2539 & 765 & 254 & 28.6 & 5.9 & Cyto & $\begin{array}{l}\text { Gohir.A12G082500/ } \\
\text { GH_A12G0712 }\end{array}$ & $100 / 100$ \\
\hline GhCYP-37 & Gh_A13G0333 & 1200 & 399 & 44.6 & 6.4 & Cyto & No found/GH_A13G0375 & $-/ 100$ \\
\hline GhCYP-38 & Gh_A13G0846 & 525 & 174 & 18.8 & 7.8 & Cyto & $\begin{array}{l}\text { Gohir.A13G103900/ } \\
\text { GH_A13G1226 }\end{array}$ & $100 / 100$ \\
\hline GhCYP-39 & Gh_D01G0026 & 675 & 224 & 24.6 & 7.3 & Cyto & $\begin{array}{l}\text { Gohir.D01G002600/ } \\
\text { GH_D01G0028 }\end{array}$ & $100 / 100$ \\
\hline GhCYP-40 & Gh_D01G0030 & 516 & 171 & 18.2 & 7.4 & Cyto & $\begin{array}{l}\text { Gohir.D01G003100/ } \\
\text { GH_D01G0033 }\end{array}$ & 99/99 \\
\hline GhCYP-41 & Gh_D01G0206 & 780 & 259 & 28.1 & 10.3 & Chlo & $\begin{array}{l}\text { Gohir.D01G020500/ } \\
\text { GH_D01G0221 }\end{array}$ & $100 / 100$ \\
\hline GhCYP-42 & Gh_D01G1605 & 522 & 173 & 18.3 & 8.5 & Cyto & $\begin{array}{l}\text { Gohir.D01G156100/ } \\
\text { GH_D01G1877 }\end{array}$ & $100 / 100$ \\
\hline GhCYP-43 & Gh_D02G0593 & 705 & 234 & 26.3 & 9.8 & Mito & $\begin{array}{l}\text { Gohir.D02G059200/ } \\
\text { GH_D02G0586 }\end{array}$ & $100 / 100$ \\
\hline GhCYP-44 & Gh_D02G1247 & 750 & 249 & 28.4 & 8.1 & Chlo & $\begin{array}{l}\text { Gohir.D02G124600/ } \\
\text { GH_D02G1353 }\end{array}$ & $98 / 100$ \\
\hline GhCYP-45 & Gh_D02G2108 & 1233 & 410 & 46.4 & 8.6 & Cyto & $\begin{array}{l}\text { Gohir.D02G212200/ } \\
\text { GH_D02G2325 }\end{array}$ & $100 / 99$ \\
\hline GhCYP-46 & Gh_D03G0186 & 1086 & 361 & 40.6 & 5.5 & Cyto & $\begin{array}{l}\text { Gohir.D03G020200/ } \\
\text { GH_D03G0207 }\end{array}$ & 99/99 \\
\hline GhCYP-47 & Gh_D03G1033 & 966 & 321 & 35.0 & 8.2 & Chlo & $\begin{array}{l}\text { Gohir.D03G108800/ } \\
\text { GH_D03G1247 }\end{array}$ & $100 / 100$ \\
\hline GhCYP-48 & Gh_D04G1620 & 522 & 173 & 18.5 & 9.6 & Cyto & $\begin{array}{l}\text { Gohir.D04G164700/ } \\
\text { GH_D04G1813 }\end{array}$ & 100/100 \\
\hline GhCYP-49 & Gh_D04G1621 & 522 & 173 & 18.3 & 8.2 & Cyto & $\begin{array}{l}\text { Gohir.D04G164800/ } \\
\text { GH_D04G1814 }\end{array}$ & $100 / 100$ \\
\hline GhCYP-50 & Gh_D04G1937 & 570 & 189 & 20.5 & 8.2 & Cyto & $\begin{array}{l}\text { Gohir.D04G016300/ } \\
\text { GH_D04G0183 }\end{array}$ & $100 / 100$ \\
\hline GhCYP-51 & Gh_D05G0033 & 1836 & 611 & 70.6 & 6.0 & Nucl & $\begin{array}{l}\text { Gohir.D05G003700/ } \\
\text { GH_D05G0030 }\end{array}$ & $100 / 100$ \\
\hline GhCYP-52 & Gh_D06G0456 & 870 & 289 & 31.9 & 7.3 & Extra & $\begin{array}{l}\text { Gohir.D06G049100/ } \\
\text { GH_D06G0504 }\end{array}$ & $100 / 100$ \\
\hline GhCYP-53 & Gh_D06G2331 & 1491 & 496 & 55.9 & 8.1 & Nucl & $\begin{array}{l}\text { Gohir.D06G084800/ } \\
\text { GH_D06G0925 }\end{array}$ & $100 / 100$ \\
\hline GhCYP-54 & Gh_D07G0381 & 2367 & 788 & 89.4 & 12.0 & Nucl & $\begin{array}{l}\text { Gohir.D07G042100/ } \\
\text { GH_D07G0441 }\end{array}$ & $100 / 99$ \\
\hline GhCYP-55 & Gh_D07G0382 & 2406 & 801 & 90.5 & 11.5 & Nucl & $\begin{array}{l}\text { Gohir.D07G042200/ } \\
\text { GH_D07G0442 }\end{array}$ & 98/97 \\
\hline
\end{tabular}


Table 1 The list of the putative CYP genes identified in G. hirsutum (Continued)

\begin{tabular}{|c|c|c|c|c|c|c|c|c|}
\hline $\begin{array}{l}\text { Gene } \\
\text { Name }\end{array}$ & Gene ID (NAU1) & $\begin{array}{l}\text { CDS } \\
\text { (bp) }\end{array}$ & $\begin{array}{l}\text { Protein } \\
\text { (aa) }\end{array}$ & $\begin{array}{l}\mathrm{MW} \\
(\mathrm{kDa})\end{array}$ & $\mathrm{pl}$ & $\begin{array}{l}\text { Subcellular } \\
\text { location }\end{array}$ & Gene ID (JGI/NAU2) & $\begin{array}{l}\text { Identity (\%) (JGI/ } \\
\text { NAU2) }\end{array}$ \\
\hline GhCYP-56 & Gh_D07G1064 & 1866 & 621 & 70.0 & 7.1 & Cyto & $\begin{array}{l}\text { Gohir.D07G112300/ } \\
\text { GH_D07G1170 }\end{array}$ & $100 / 100$ \\
\hline GhCYP-57 & Gh_D07G2233 & 1359 & 452 & 49.8 & 4.9 & Chlo & $\begin{array}{l}\text { Gohir.D07G226300/ } \\
\text { GH_D07G2429 }\end{array}$ & 100/99 \\
\hline GhCYP-58 & Gh_D08G0452 & 711 & 236 & 26.7 & 9.5 & Mito/Nucl & $\begin{array}{l}\text { Gohir.D08G050300/ } \\
\text { GH_D08G0461 }\end{array}$ & $100 / 100$ \\
\hline GhCYP-59 & Gh_D08G1359 & 768 & 255 & 27.5 & 8.4 & Chlo & $\begin{array}{l}\text { Gohir.D08G143400/ } \\
\text { GH_D08G1489 }\end{array}$ & $89 / 88$ \\
\hline GhCYP-60 & Gh_D08G1477 & 483 & 160 & 17.4 & 7.8 & Cyto & $\begin{array}{l}\text { Gohir.D08G154400/ } \\
\text { GH_D08G1612 }\end{array}$ & 99/99 \\
\hline GhCYP-61 & Gh_D08G1766 & 1032 & 343 & 38.0 & 5.5 & Extra & $\begin{array}{l}\text { Gohir.D08G182600/ } \\
\text { GH_D08G1902 }\end{array}$ & $100 / 100$ \\
\hline GhCYP-62 & Gh_D08G2018 & 1965 & 654 & 72.1 & 11.3 & Nucl & $\begin{array}{l}\text { Gohir.D08G205800/ } \\
\text { GH_D08G2160 }\end{array}$ & 100/99 \\
\hline GhCYP-63 & Gh_D09G0253 & 1311 & 436 & 48.1 & 4.7 & Cyto & $\begin{array}{l}\text { Gohir.D09G025500/ } \\
\text { GH_D09G0307 }\end{array}$ & $100 / 100$ \\
\hline GhCYP-64 & Gh_D09G1874 & 1032 & 343 & 37.8 & 5.5 & Extra & $\begin{array}{l}\text { Gohir.D09G191800/ } \\
\text { GH_D09G2069 }\end{array}$ & $100 / 100$ \\
\hline GhCYP-65 & Gh_D10G0925 & 1089 & 362 & 40.3 & 6.9 & Cyto & $\begin{array}{l}\text { Gohir.D10G095600/ } \\
\text { GH_D10G1032 }\end{array}$ & $100 / 100$ \\
\hline GhCYP-66 & Gh_D10G1953 & 576 & 191 & 20.6 & 8.2 & Cyto & $\begin{array}{l}\text { Gohir.D10G195800/ } \\
\text { GH_D10G2172 }\end{array}$ & $100 / 100$ \\
\hline GhCYP-67 & Gh_D10G2442 & 666 & 221 & 23.8 & 9.4 & Cyto & $\begin{array}{l}\text { Gohir.D10G248100/ } \\
\text { GH_D10G2719 }\end{array}$ & $100 / 100$ \\
\hline GhCYP-68 & Gh_D11G0793 & 996 & 331 & 37.2 & 5.5 & Extra & $\begin{array}{l}\text { Gohir.D11G079500/ } \\
\text { GH_D11G0807 }\end{array}$ & $100 / 100$ \\
\hline GhCYP-69 & Gh_D11G1133 & 705 & 234 & 26.7 & 8.9 & Mito/Nucl & $\begin{array}{l}\text { Gohir.D11G113000/ } \\
\text { GH_D11G1161 }\end{array}$ & $100 / 100$ \\
\hline GhCYP-70 & Gh_D12G0709 & 210 & 69 & 7.5 & 9.1 & Cyto/Nucl & $\begin{array}{l}\text { Gohir.D06G090500/ } \\
\text { GH_D06G0997 }\end{array}$ & $98 / 94$ \\
\hline GhCYP-71 & Gh_D12G0724 & 1791 & 596 & 65.4 & 8.2 & Cyto/Nucl & $\begin{array}{l}\text { Gohir.D12G075500/ } \\
\text { GH_D12G0928 }\end{array}$ & $100 / 100$ \\
\hline GhCYP-72 & Gh_D12G0852 & 810 & 269 & 30.5 & 6.5 & Cyto & Gohir.D12G089100/No found & 99/- \\
\hline GhCYP-73 & Gh_D12G2822 & 519 & 172 & 18.1 & 8.5 & Cyto & $\begin{array}{l}\text { Gohir.D12G033300/ } \\
\text { GH_D12G0323 }\end{array}$ & $100 / 100$ \\
\hline GhCYP-74 & Gh_D13G0372 & 1212 & 403 & 45.4 & 7.2 & Cyto & $\begin{array}{l}\text { Gohir.D13G036300/ } \\
\text { GH_D13G0367 }\end{array}$ & $100 / 100$ \\
\hline GhCYP-75 & Gh_D13G1093 & 525 & 174 & 18.8 & 7.4 & Cyto & $\begin{array}{l}\text { Gohir.D13G107100/ } \\
\text { GH_D13G1170 }\end{array}$ & $100 / 100$ \\
\hline GhCYP-76 & Gh_Sca004717G03 & 1377 & 458 & 49.9 & 6.8 & Chlo & $\begin{array}{l}\text { Gohir.D11G293200/ } \\
\text { GH_A11G3184 }\end{array}$ & 100/99 \\
\hline GhCYP-77 & Gh_Sca004717G12 & 1377 & 458 & 49.9 & 5.9 & Chlo & $\begin{array}{l}\text { Gohir.D11G296300/ } \\
\text { GH_D11G3210 }\end{array}$ & $100 / 100$ \\
\hline GhCYP-78 & Gh_Sca004880G02 & 780 & 259 & 28.1 & 10.5 & Chlo & $\begin{array}{l}\text { Gohir.A01G022000/ } \\
\text { GH_A01G0230 }\end{array}$ & 100/99 \\
\hline GhCYP-79 & Gh_Sca006066G02 & 513 & 170 & 18.6 & 9.4 & Cyto/Nucl & $\begin{array}{l}\text { Gohir.D01G194000/ } \\
\text { GH_D01G2301 }\end{array}$ & $100 / 100$ \\
\hline
\end{tabular}

samples were identified as potentially expressed transcripts. We focused on the significantly differentially expressed genes (fold change $[\mathrm{FC}]>2$ or $\mathrm{FC}<0.5$ ) in the various stresses. GhCYPs that showed significant differential expression at least one treatment time were listed in Fig. 3. In the cold treatment, 14 GhCYPs were commonly down-regulated significantly apart from GhCYP-49 at 1 hct (hours after cold treated) and 3 hct and GhCYP-52 at 6 hct, which showed up-regulated significantly. At 1 hht (hours after hot treated), 22 of 26 

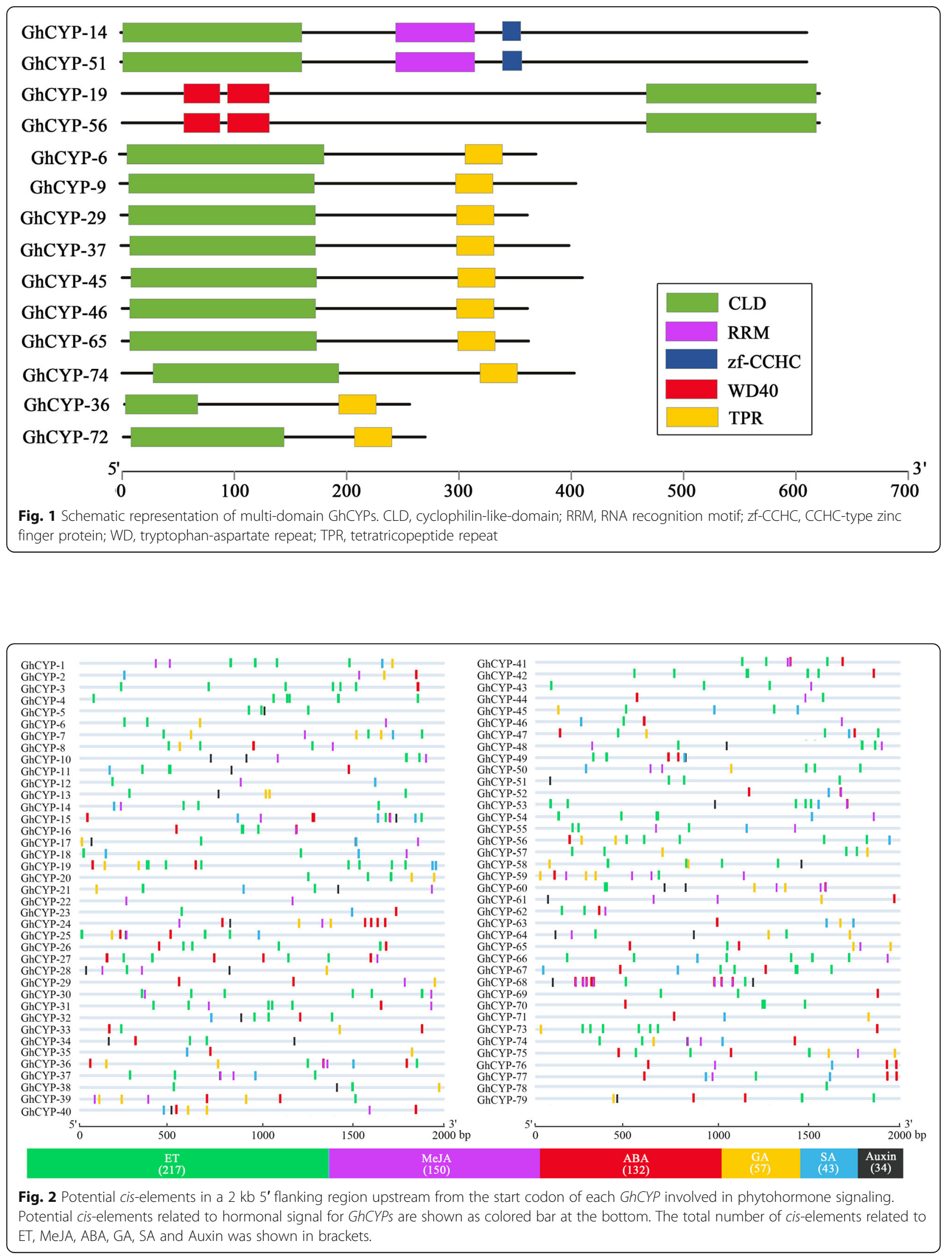


\begin{tabular}{|c|c|c|c|c|c|c|c|c|c|}
\hline Cold & 1 hct & 3 hct & 6 hct & 12 hct & Hot & $1 \mathrm{hht}$ & $3 \mathrm{hht}$ & $6 \mathrm{hht}$ & $12 \mathrm{hht}$ \\
\hline GhCYP-3 & 0.36 & 0.12 & 0.09 & 0.95 & GhCYP-3 & 1.02 & 0.58 & 1.01 & 2.49 \\
\hline GhCYP-15 & 0.52 & 0.93 & 1.41 & 0.11 & GhCYP-7 & 0.37 & 0.89 & 0.85 & 1.28 \\
\hline GhCYP-20 & 0.42 & 0.72 & 0.85 & 1.48 & GhCYP-8 & 0.38 & 1.18 & 1.56 & 1.05 \\
\hline GhCYP-24 & 1.10 & 0.74 & 0.90 & 0.37 & GhCYP-15 & 0.36 & 0.54 & 0.88 & 0.79 \\
\hline GhCYP-25 & 0.87 & 0.48 & 0.76 & 0.30 & GhCYP-20 & 0.34 & 1.59 & 0.85 & 2.37 \\
\hline GhCYP-27 & 0.66 & 1.66 & 0.30 & 0.30 & GhCYP-22 & 0.42 & 0.78 & 0.78 & 1.76 \\
\hline GhCYP-42 & 0.52 & 0.38 & 0.17 & 1.15 & GhCYP-24 & 1.08 & 1.67 & 3.08 & 1.14 \\
\hline GhCYP-49 & 2.79 & 2.54 & 1.05 & 1.08 & GhCYP-27 & 1.24 & 2.51 & 1.11 & 1.81 \\
\hline GhCYP-52 & 0.34 & 0.74 & 2.51 & 0.78 & GhCYP-34 & 0.39 & 1.06 & 1.39 & 1.19 \\
\hline GhCYP-54 & 0.64 & 0.15 & 0.00 & 0.22 & GhCYP-38 & 0.60 & 1.21 & 0.92 & 3.85 \\
\hline GhCYP-73 & 0.56 & 1.15 & 0.51 & 0.35 & GhCYP-39 & 0.49 & 1.26 & 0.82 & 0.97 \\
\hline GhCYP-75 & 0.47 & 0.40 & 0.41 & 0.18 & GhCYP-41 & 0.36 & 1.45 & 0.92 & 3.64 \\
\hline GhCYP-76 & 0.39 & 0.74 & 1.19 & 1.94 & GhCYP-42 & 0.85 & 0.91 & 1.14 & 3.01 \\
\hline GhCYP-77 & 0.48 & 0.86 & 0.38 & 1.38 & GhCYP-44 & 0.25 & 0.87 & 1.27 & 0.83 \\
\hline & & & & & GhCYP-47 & 0.38 & 0.61 & 0.87 & 1.27 \\
\hline Salt & $1 \mathrm{hst}$ & $3 \mathrm{hst}$ & $6 \mathrm{hst}$ & $12 \mathrm{hst}$ & GhCYP-49 & 2.25 & 1.10 & 0.74 & 1.15 \\
\hline GhCYP-3 & 2.63 & 1.20 & 0.94 & 1.51 & GhCYP-52 & 0.37 & 0.62 & 1.32 & 0.96 \\
\hline GhCYP-7 & 0.67 & 0.75 & 0.42 & 0.61 & GhCYP-54 & 0.48 & 1.45 & 1.63 & 0.76 \\
\hline GhCYP-20 & 0.50 & 0.96 & 0.62 & 0.89 & GhCYP-57 & 0.00 & 3.12 & 0.79 & 0.89 \\
\hline GhCYP-22 & 0.79 & 0.83 & 0.45 & 1.25 & GhCYP-58 & 0.44 & 1.49 & 0.82 & 0.81 \\
\hline GhCYP-24 & 1.13 & 0.75 & 2.13 & 1.12 & GhCYP-59 & 0.43 & 0.79 & 0.63 & 1.55 \\
\hline GhCYP-38 & 0.72 & 0.43 & 0.47 & 2.75 & GhCYP-61 & 3.52 & 4.39 & 5.03 & 1.18 \\
\hline GhCYP-41 & 0.64 & 0.83 & 0.32 & 1.37 & GhCYP-69 & 0.38 & 1.64 & 2.22 & 1.30 \\
\hline GhCYP-42 & 2.11 & 1.47 & 1.05 & 1.94 & GhCYP-76 & 0.31 & 1.76 & 0.66 & 1.66 \\
\hline GhCYP-47 & 1.20 & 0.85 & 0.47 & 0.55 & GhCYP-77 & 0.29 & 1.22 & 0.47 & 0.87 \\
\hline GhCYP-49 & 4.44 & 1.34 & 1.59 & 1.25 & GhCYP-78 & 0.32 & 1.60 & 1.33 & 2.74 \\
\hline GhCYP-54 & 0.96 & 0.14 & 1.81 & 0.37 & & & & & \\
\hline GhCYP-57 & 0.72 & 2.72 & 0.67 & 0.66 & PEG & $1 \mathrm{hpt}$ & $3 \mathrm{hpt}$ & $6 \mathrm{hpt}$ & $12 \mathrm{hpt}$ \\
\hline GhCYP-61 & 1.92 & 1.03 & 2.75 & 1.24 & GhCYP-3 & 2.76 & 1.38 & 0.85 & 2.15 \\
\hline GhCYP-75 & 0.76 & 0.40 & 0.84 & 1.35 & GhCYP-38 & 0.85 & 0.40 & 0.26 & 3.99 \\
\hline GhCYP-76 & 0.48 & 1.36 & 0.50 & 1.35 & GhCYP-41 & 0.79 & 0.55 & 0.46 & 1.39 \\
\hline GhCYP-77 & 0.47 & 0.95 & 0.32 & 0.47 & GhCYP-42 & 1.66 & 1.21 & 0.85 & 2.12 \\
\hline GhCYP-78 & 0.53 & 0.97 & 0.46 & 0.99 & GhCYP-47 & 0.52 & 0.83 & 0.90 & 0.38 \\
\hline \multirow[t]{3}{*}{ Low } & & & \multirow{2}{*}{\multicolumn{2}{|c|}{ High }} & GhCYP-49 & 2.81 & 0.80 & 1.36 & 1.25 \\
\hline & \multirow{2}{*}{\multicolumn{4}{|c|}{ Foldchange }} & GhCYP-73 & 1.81 & 1.14 & 0.69 & 2.08 \\
\hline & & & & & GhCYP-75 & 1.39 & 0.45 & 0.81 & 1.80 \\
\hline \multicolumn{10}{|c|}{$\begin{array}{l}\text { Fig. } 3 \text { Expression profiles of GhCYPs in response to different abiotic stress. The fold change (FC) is the ratio of treatment FPKM to control FPKM } \\
\text { Red color indicates higher up-regulation expression. Blue color indicates lower down-regulation expression. The significantly differentially } \\
\text { expressed genes were FC }>2 \text { (up-regulated) or FC }<0.5 \text { (down-regulated). hct, hours cold treatment; hht, hours hot treatment; hst, hours salt } \\
\text { treatment; hpt, hours PEG treatment }\end{array}$} \\
\hline
\end{tabular}

GhCYPs are response to hot treatment. Of these, only the expression level of GhCYP-49 and GhCYP-61 was up-regulated significantly. At 3 hht, 6 hht and 12 hht, only 12 GhCYPs exhibited differential expression pattern.. Of these, up to 11 genes were up-regulated. Most GhCYP genes were found to be down-regulated under the condition of salt treatment apart from GhCYP-3, GhCYP-24, GhCYP-38, GhCYP-42, GhCYP-49, GhCYP57 and GhCYP-61. After PEG (polyethylene glycol) treatment, only 8 GhCYPs displayed differential expression pattern, of which, GhCYP-3, GhCYP-42, GhCYP-49 and GhCYP-73 were up-regulated, GhCYP-41, GhCYP-47 
and GhCYP-75 were down-regulated. Only the expression of $G$ hCYP-38 showed be down-regulated at $3 \mathrm{hpt}$ (hours after PEG treated) and $6 \mathrm{hpt}$, and then be upregulated at $12 \mathrm{hpt}$. These expression patterns suggest that CYPs undertake multiple functions to help the cotton counter various complex environmental challenges.

\section{Expression profiles of GhCYPs under the stress of $V$. dahliae}

To gain a better understanding of the roles of CYP family genes in the cotton resistance against $V$. dahliae, the expression profiles were obtained by RNA-Seq. A total of 30 GhCYP genes showed differential expression at least one time point (hpi, hours post inoculation), including 19 upregulated genes and 11 downregulated genes (Fig. 4). Notably, levels of GhCYP-10, GhCYP-22, GhCYP-48 and GhCYP-59 were up-regulated at all five treatment time points. Additionally, the expression of GhCYP-11, GhCYP-27, GhCYP-37, GhCYP-45 and GhCYP-74 was down-regulated in most time points. These results revealed that GhCYPs were associated with the interaction between cotton and $V$. dahliae.

\section{GhCYP-3 contains conserved amino acid residues and has PPlase activity}

GhCYP-3, cloned from G. hirsutum cv. JM20, contains a single cyclophilin domain, 173 amino acid residues with a calculated molecular mass of $18.2 \mathrm{kDa}$ and a pI of 8.34. Alignment with previously characterized Arabidopsis and human CYP (AtCYP19-1 and hCypA) revealed that GhCYP-3 contains seven conserved amino acid residues that critically affect PPIase activity (Fig. 5a). GhCYP-3 was found to be expressed in root, stem and leaf of unchallenged cotton plants with $V$. dalihae (Fig. 5b). Translation fusion of GhCYP-3 with GFP was constructed under the control of $35 \mathrm{~S}$ promoter, and then transiently expressed in onion epidermal cells. Fluorescent imaging of the GhCYP-3-GFP bombarded onion epidermal cells showed both cytoplasm and nuclear localization (Fig. 5c).

The GhCYP-3 ORF was cloned into pET-32a at SacI and BglII sites, thus the recombinant plasmid was constructed (Fig. 6a). SDS-PAGE and western blot analysis revealed that the fusion TrxA- $6 \times$ His-S-tag- $6 \times$ His (THS, $20.4 \mathrm{kDa}$; empty vector as control) and TrxA-6 $\times$ His-Stag-GhCYP3 (THS-CYP, $34.6 \mathrm{kDa}$ ) proteins were highly expressed in the E. coli BL21(DE3) at $37^{\circ} \mathrm{C}$ with $1 \mathrm{mM}$ IPTG for $4 \mathrm{~h}$ (Fig. 6b). The recombinant THS-CYP protein was purificated using the nickel-affinity. Further, we measured the PPIase activity of the recombinant protein by a coupled assay using synthetic peptide synthetic peptide succinyl- Ala-Ala-Pro-Phe-p-nitroanilide. The isomerization of the peptide substrate was observed in the presence of recombinant GhCYP-3, showing OD values higher than that of the blank, a negative control (Fig. 6c). These results indicated that GhCYP-3 has PPIase activity in vitro.

\section{GhCYP-3 expression is upregulated in response to $V$. dahliae invasion}

To analyze the expression pattern of GhCYP-3 in three cotton cultivars with different degrees of resistance to $V$. dahliae, the roots of two-week-old cotton seedlings were sampled at $0,4,8,12,24$ and $48 \mathrm{hpi}$. The qRT-PCR results revealed that the transcriptional levels of GhCYP-3, compared with $0 \mathrm{hpi}$, were significantly upregulated in two-resistant cultivars Pima90-53 and JM-20 at 4 hpi. However, at the same inoculation time, GhCYP-3 in susceptible Han-208 was strongly downregulated and reached the highest expression level until 24 hpi (Fig 7). These results suggested that GhCYP-3 is upregulated and earlier involved in the cotton interaction with $V$. dahliae in resistant than in susceptible cultivars.

\section{Overexpression of GhCYP-3 in Arabidopsis improves plant Verticillium wilt resistance}

To further evaluate whether the GhCYP-3 functions in plant resistance to Verticillium wilt, we designed primers to amplify the ORF of GhCYP-3 into the plant expression vector pBI121 (Fig. 8a), which will make GhCYP-3 overexpression in transgenic Arabidopsis plants. By selection on kanamycin-containing medium (Fig. 8b), ten potential $T_{1}$ transgenic lines were generated. After PCRverification of gene insertion (Fig. 8c), two lines of the $\mathrm{T}_{3}$ generation displaying GhCYP-3 overexpression (Fig. 8d) were selected for analysis of PPIase activity and Verticillium wilt resistance.

Under unstressed conditions (treated with $\mathrm{H}_{2} \mathrm{O}$ ), no significant PPIase activity was enhanced in two GhCYP3-expressing transgenic lines compared to the WT (wild type). However, these two transgenic lines, infected with $V$. dahliae for $3 \mathrm{~d}$, showed increased PPIase activity of $0.51 \pm 0.03(\mathrm{~L} 1)$ and $0.45 \pm 0.03 \mathrm{nmol} \cdot \mathrm{s}^{-1} \cdot \mathrm{mg}^{-1}$ protein (L2) compared to $0.35 \pm 0.03 \mathrm{nmol} \cdot \mathrm{s}^{-1} \cdot \mathrm{mg}^{-1}$ protein observed in the WT (Fig. 8e).

In response to $V$. dahliae infection, two independent overexpressing lines displayed less wilting and smaller degree of leaf etiolation in comparison with WT plants at 15 dpi (Fig. 9a). Disease evaluation further indicated that the disease indices of transgenic lines were significantly lower than that of the WT (Fig. 9b). Furthermore, we examined the colonization in stems of infected WT and transgenic plants by isolation and cultivation of $V$. dahliae on PDA (potato dextrose agar) for 8 days. As a result, less fungal colonies came out from transgenic plants comparing to WT (Fig. 9c and d). These fungi recovery assay indicated that GhCYP-3 has a specialized effect of growth inhibition on $V$. dahliae. 


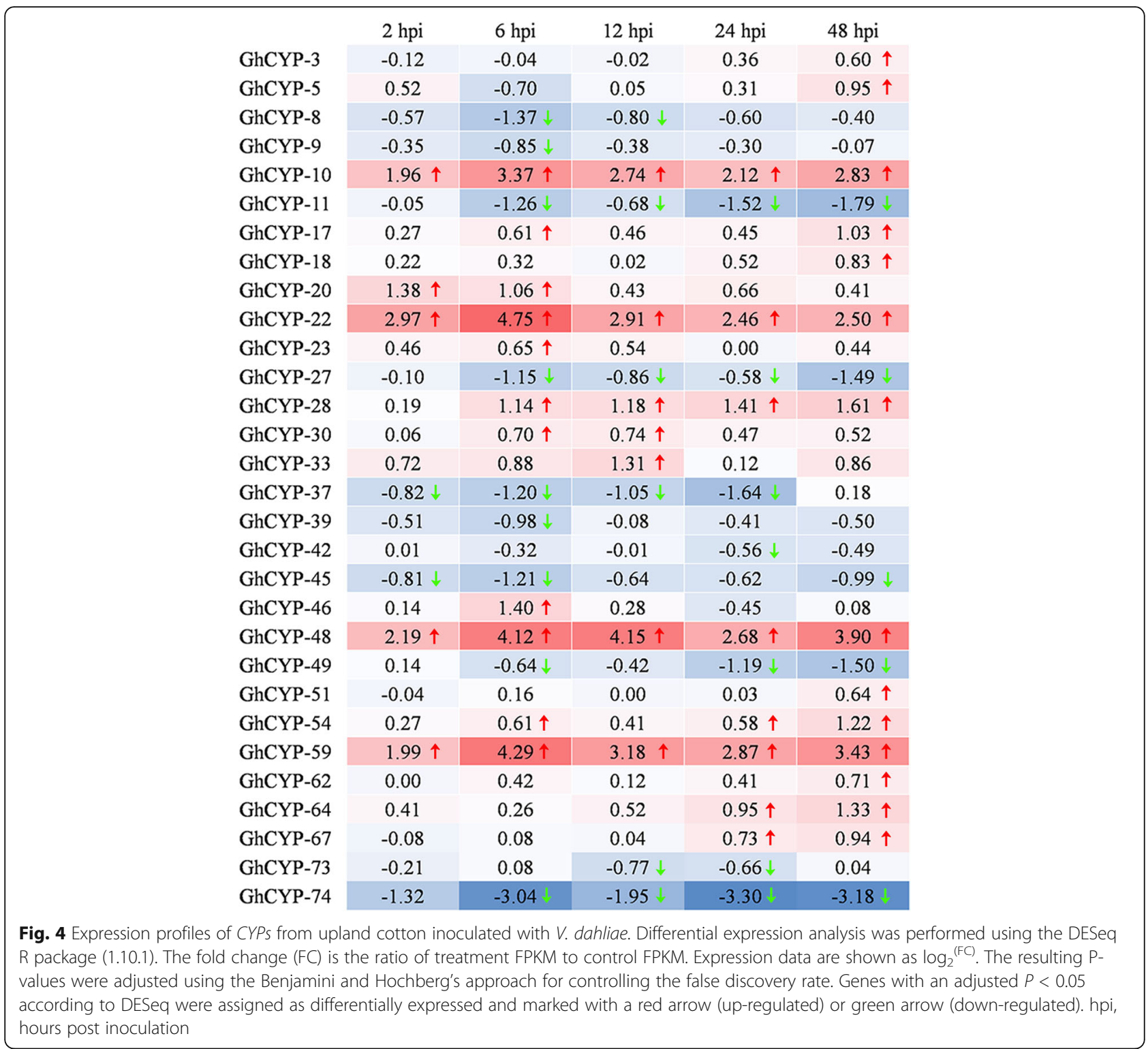

\section{GhCYP-3 exhibited obvious inhibitory effects on $V$. dahliae}

We further assessed the inhibitory effects of GhCYP-3 on $V$. dahliae. As showed in Fig. 10a, distinct inhibition zones formed around the disc containing recombinant GhCYP-3. Additionally, $V$. dahliae spores were inoculated with plant extracts from WT and transgenic lines and then be spread on PDA plates. After $48 \mathrm{~h}$, extracts from all the plants significantly reduced the number of colonies compared to the $\mathrm{H}_{2} \mathrm{O}$ (as control). However, the number of fungal colonies on plates contain transgenes extracts was significantly less than the WT (Fig. 10b and c). These results indicated that GhCYP-3 can efficiently inhibit conidia germinating and hyphae growth of $V$. dahliae in vitro.

\section{Discussion}

CYP genes family has been systematically analyzed in several plants, such as Arabidopsis [8], rice [22] and soybean [10]. Recently, 78 CYP genes were also identified in G. hirsutum L. acc. TM-1 from Illumina paired-end genomic sequencing (NAU version 1.1 ) by Chen et al. [23]. Now, NAU version 2.1, highly accurate reference grade genome assemblies and annotations for G. hirsutum, was generated [21]. A fairly large number of gaps and erroneous assemblies were successfully filled and corrected in new NAU version 2.1 [21]. Therefore, in the present study we identified a total of 79 CYP genes by integrating NAU version 1.1, JGI database and NAU version 2.1 (Table 1). Compared to the previous report from Chen et al., the supplementary three CYPs (GhCYP-49, GhCYP- 


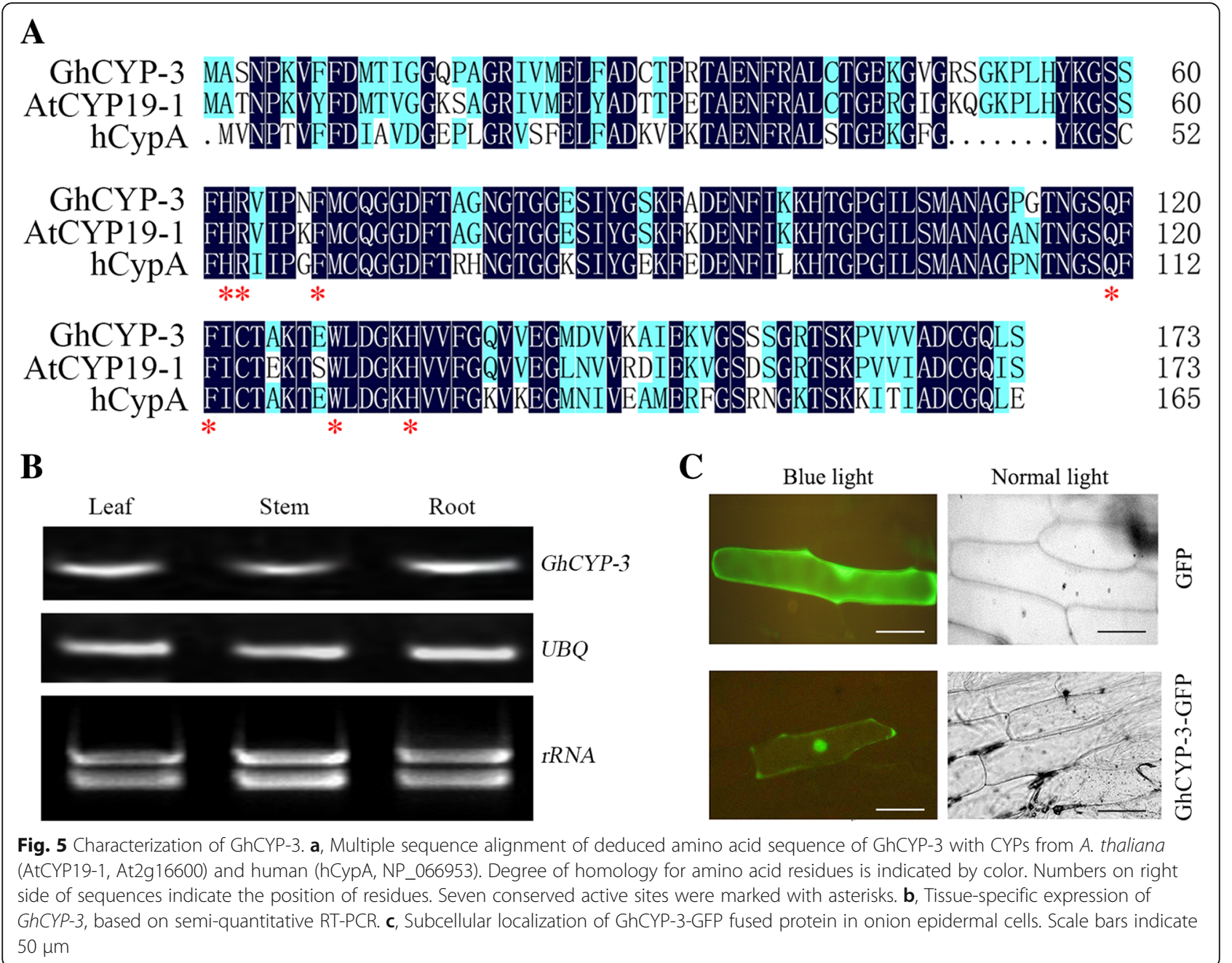

60 and GhCYP-70) were identified in this study. Besides, two CYPs (Gh_Sca140771G01 and Gh_A12G1281), undiscoverable in JGI database and NAU version 2.1, were removed. It thus made the candidate CYP genes in G. hirsutum more reliable.

All 79 CYPs in G. hirsutum have conservative PPIase domain (CLD). Of which, 14 GhCYPs are multi-domain proteins (Fig. 1). In addition to the CLD, GhCYP-14 and GhCYP-51 contain RRM and zf-CCHC. The RRM domain was found in proteins involved in RNA processing where it mediates binding to various RNAs to execute both housekeeping functions and regulatory mechanisms [24]. Proteins that contain zinc fingers typically interact with DNA and RNA, and serve primarily to alter the binding specificity of a particular protein. In addition, GhCYP14 and GhCYP-51 were predicted to be localized in the nuclear (Table 1). Therefore, these two CYPs possibly mediate ribosomal association while the CLD catalyzes peptidyl prolyl cis-trans isomerization of nascent polypeptides. GhCYP-19 and GhCYP-56 contain WD40 domain that generally serve as a rigid scaffold for protein interactions
[25]. Other 10 multi-domain GhCYPs contain TPR domain, which mainly act as interactive scaffolds in the formation of protein complexes and regulators of RNA metabolism involved in the immune response [26, 27].

Regulation of gene expression via specific cis-regulatory elements in the promoter regions has evolved as a major adaptive mechanism to respond to environmental stress in plants [28]. Phytohormones are critical to the regulation of plant development and defense [29]. Thus, the analysis of the putative cis-regulatory elements relating to hormone helps to advance our understanding of GhCYPs involving stress tolerance in cotton. Six hormones (ET, MeJA, ABA, GA, SA and Auxin) responsive regulatory elements were detected in the potential promoter regions of GhCYPs (Fig. 2), indicating that GhCYPs involve different hormone-mediated signaling pathways.

In 2010, we isolated 203 ESTs from a cDNA library using suppression subtractive hybridization ( $\mathrm{SSH}$ ) with a resistant upland cotton cultivar Jimian20 induced with $V$. dahliae. Of which, an EST encoded a partial polypeptide with homology to CYP [30]. This is the first report 

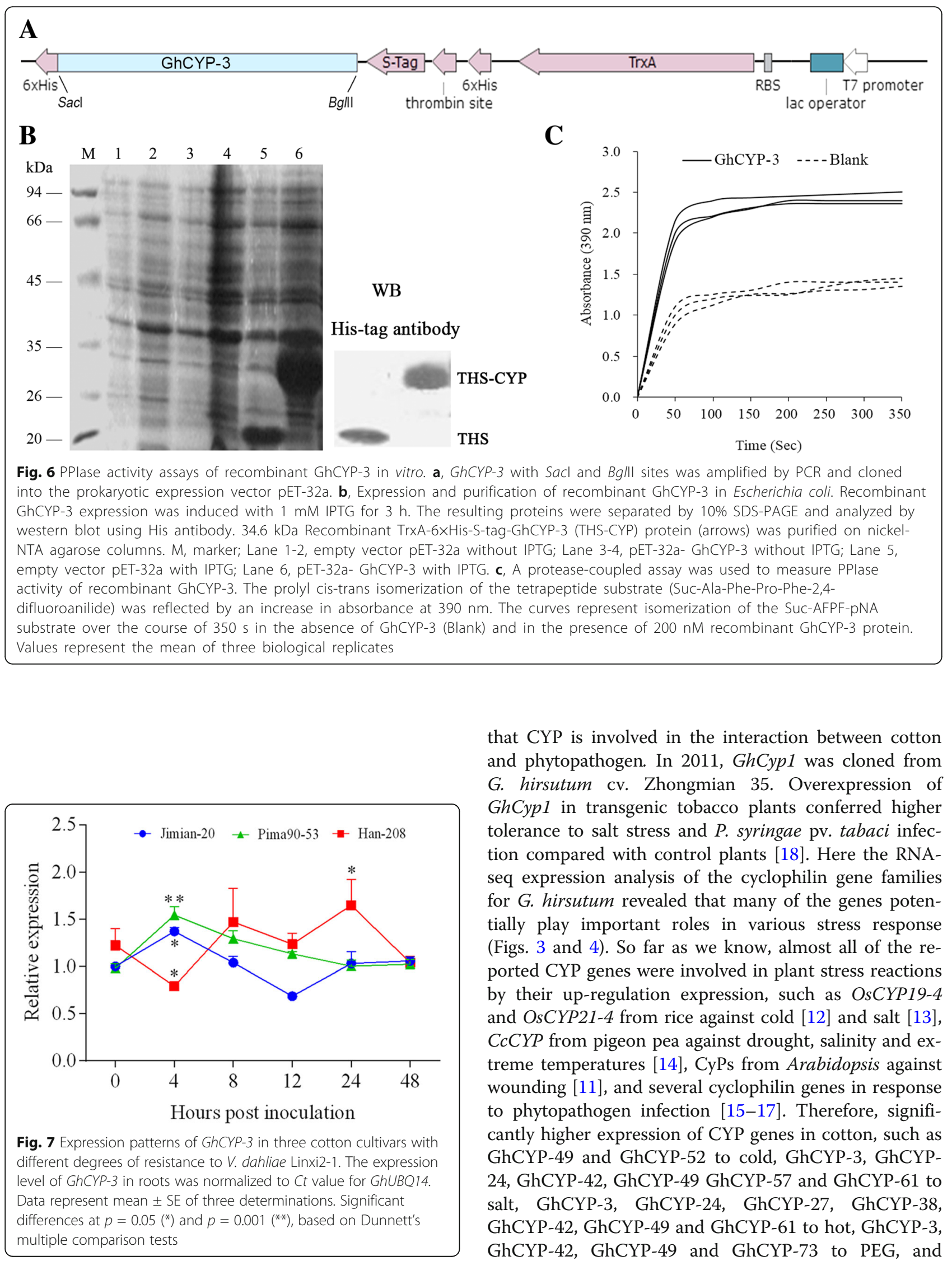

that CYP is involved in the interaction between cotton and phytopathogen. In 2011, GhCyp1 was cloned from G. hirsutum cv. Zhongmian 35. Overexpression of GhCyp1 in transgenic tobacco plants conferred higher tolerance to salt stress and $P$. syringae pv. tabaci infection compared with control plants [18]. Here the RNAseq expression analysis of the cyclophilin gene families for G. hirsutum revealed that many of the genes potentially play important roles in various stress response (Figs. 3 and 4). So far as we know, almost all of the reported CYP genes were involved in plant stress reactions by their up-regulation expression, such as OsCYP19-4 and OsCYP21-4 from rice against cold [12] and salt [13], ${ }_{c} C Y P$ from pigeon pea against drought, salinity and extreme temperatures [14], CyPs from Arabidopsis against wounding [11], and several cyclophilin genes in response to phytopathogen infection [15-17]. Therefore, significantly higher expression of CYP genes in cotton, such as GhCYP-49 and GhCYP-52 to cold, GhCYP-3, GhCYP24, GhCYP-42, GhCYP-49 GhCYP-57 and GhCYP-61 to salt, GhCYP-3, GhCYP-24, GhCYP-27, GhCYP-38, GhCYP-42, GhCYP-49 and GhCYP-61 to hot, GhCYP-3, GhCYP-42, GhCYP-49 and GhCYP-73 to PEG, and 


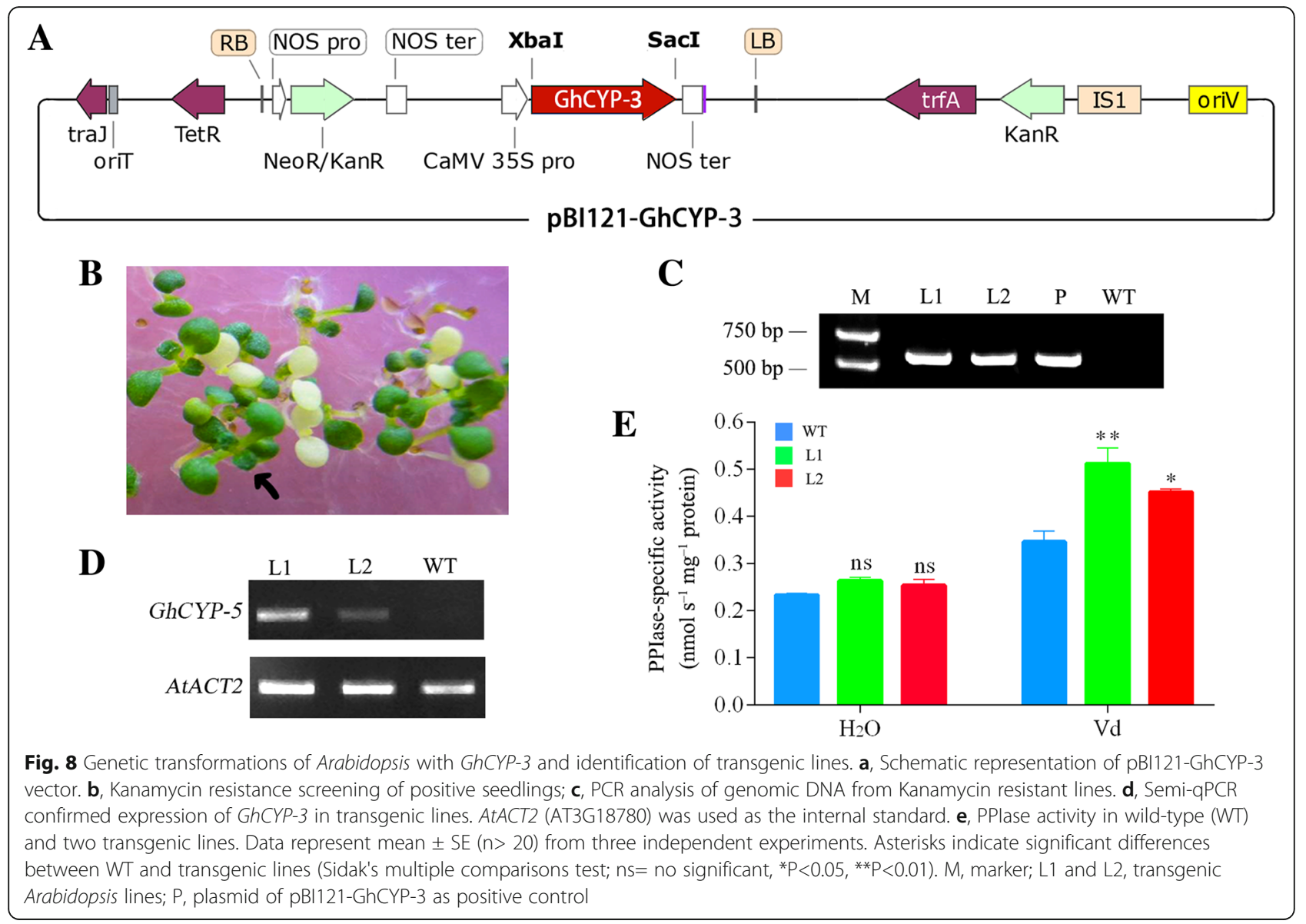

GhCYP-3, GhCYP-5, GhCYP-10, GhCYP-17, GhCYP18, GhCYP-20, GhCYP-22, GhCYP-23, GhCYP-28, GhCYP-30, GhCYP-33, GhCYP-46, GhCYP-48, GhCYP51, GhCYP-54, GhCYP-59, GhCYP-62, GhCYP-64 and GhCYP-67 to $V$. dahliae suggests likely functional importance under these stress conditions. However, further functional studies are required to unravel the precise role of these candidates during cotton response to biotic and abiotic stress conditions.

GhCYP-3 contained the above-mentioned EST sequence related to $V$. dahliae-infection and showed $98 \%$ similarity in amino acid sequences with GhCyp1. Thus, GhCYP-3 potentially plays an important role in regulating cotton immune response. The results of expression (Fig. 7) and overexpression analysis (Fig. 9) further supposed that GhCYP-3 was involved in the cotton defence to $V$. dahliae. Plant CYPs could locate in multiple cell organelles, such as ER [31], chloroplast [9], Golgi [13], cytoplasm and nucleus [32, 33]. Interestingly, the GhCYP3-GFP fusion protein was localized in the cell cytoplasm as well as the cell nucleus in onion epidermal cells (Fig. 5c). Nuclear localization was proposed to play a possible role in the regulation of gene expression [33]. GmCYP1 was as a "helper" that activates the enzymatic activity of a Phytophthora sojae RXLR effector Avr3b in a PPIase activity-dependent manner [34]. Furthermore, GmCYP1 was demonstrated to interact with the isoflavonoid regulators GmMYB176 and 14-3-3 protein, suggesting that it participates in isoflavonoids metabolism and plays role in defense [33]. AtCYP57 was proved to be involved in the $A$. thaliana response to $P$. syringae infection by influencing callose accumulation and PAD4 (peptidyl arginine deiminase type 4) expression, which interacts with EDS1 (enhanced disease susceptibility 1) to provide basal immune response in plants. Nucleus location makes authors inferred that AtCYP57 could directly regulate the translation of defence genes [32]. Therefore, we inferred that GhCYP-3 play resistance function, may like AtCYP57 and GmCYP1, in the cell nucleus by directly interacting with some transcription factor to regulate the translation of defence genes, which lead to the production of antimicrobial metabolites.

Alternatively, GhCYP-3 was also located in the cytoplasm, indicating that it needs to play some extra roles, presumably including direct antifungal activity. Antifungal activities of CYPs have been reported from some plants including ginseng [35], Chinese cabbage [36], chickpea [37] and black-eyed pea [38]. In our study, 


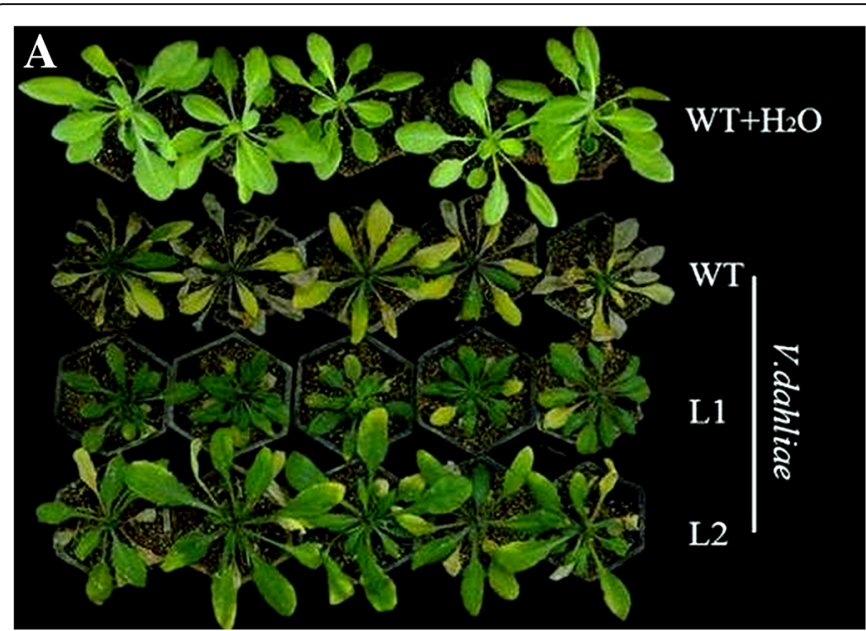

\section{B}

C

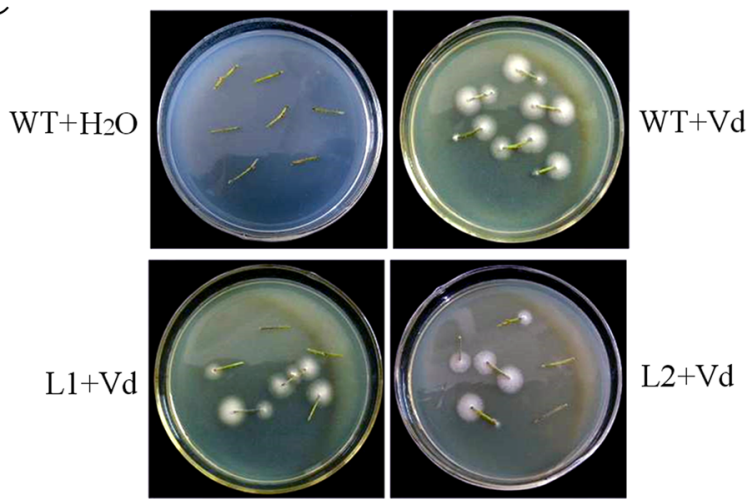

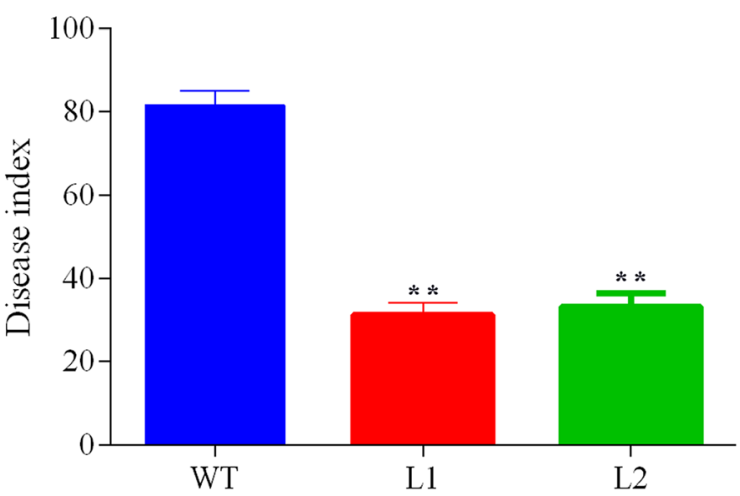

D

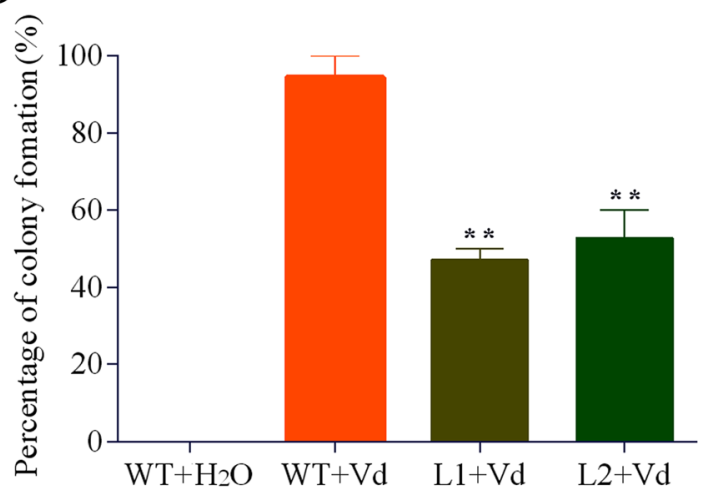

Fig. 9 Enhanced disease tolerance of Arabidopsis plants overexpressing GhCYP-3. a, Phenotype comparison of wild-type (WT) and T3 independent GhCYP-3 transgenic plants (L1 and L2) inoculated with $V$. dahliae for 15 days. b. Disease indices of the WT and transgenic plants. Error bars indicate the SE $(n>30)$ of three biological replicates. Asterisks indicate statistically significant differences as determined by Dunnett's multiple comparisons test (**P<0.01). c, 10-dpi stem sections were plated on PDA medium. Colonies were formed after incubating 7 days at 25 ${ }^{\circ} \mathrm{C} . \mathrm{Vd}, \mathrm{V}$. dahliae. $\mathbf{d}$, Percentage of colony formation. Error bars indicate the SE $(\mathrm{n}=18)$ of three biological replicates. Asterisks indicate statistically significant differences as determined by Dunnett's multiple comparisons test $\left.{ }^{* *} P<0.01\right)$

recombinant GhCYP-3 displayed evident inhibitory effects on $V$. dahliae on the plate (Fig. 10a). In a state of nature, the concentration of CYP in plant is hardly so high in the plate. Otherwise, there was no definite evidence to suggest that CYP is involved in plant resistance to pathogens by direct antifungal activity in vivo at present. However, we do not rule it out, because the extracts from GhCYP-3 transgenic Arabidopsis displayed significantly inhibit activity to conidia germinating and hyphae growth of $V$. dahliae (Fig. 10b). Many characterized antifungal proteins active on the fungal cell wall, plasma membrane and or intracellular targets [39]. For example, Buforin 2 from the stomach tissue of Bufo bufo gargarizans can translocate through the plasma membrane and exhibits antifungal activity upon interaction with fungal DNA and RNA [40]. During the infection process, $V$. dahliae forms penetration peg and specialized fungus-host interface to secret secretory effector proteins [41]. This is probably a chance for antifungal proteins including GhCYP-3 produced by host plant encountering and entering into the cytoplasm of $V$. dahliae. Additionally, we identified 10 putative CYPs (VdCYPs) in $V$. dahliae strain VdLs.17 genome with very high sequence similarity with GhCYP-3 (Additional file 3: Table S3). These mean that GhCYP-3 may replace VdCYPs and carry out the same biological function in the cytoplasm of $V$. dahliae. Thus, we inferred that the antifungal activity of GhCYP-3 has been shown to be due to the effect on the normal function of VdCYPs, which is essential for the development of $V$. dahliae. Nevertheless, further research is needed to confirm this bold deduction.

\section{Conclusions}

This is the first systematic analysis of CYP family genes in cotton aiming to help clarifying the gene sequence characteristics and expression patterns. The putative cisregulatory elements predication and expression divergence 


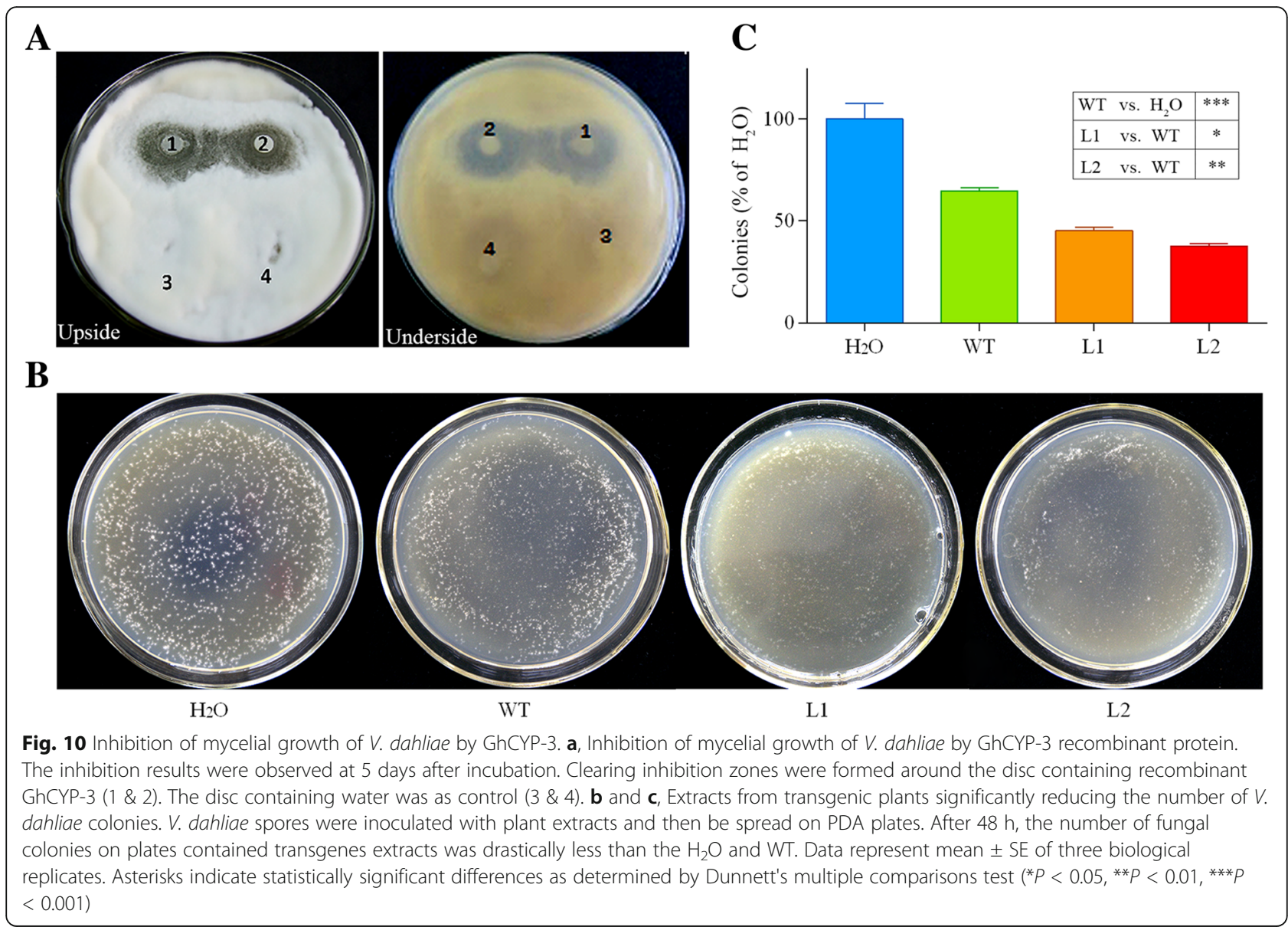

suggested that the GhCYP genes are involved in multiple phytohormone regulation pathways and responses to various abiotic stress and $V$. dahliae infection. These results will provide potential clues for the selection of candidate genes for further in-depth study on the functional characterization. Furthermore, GhCYP-3 showed both cytoplasmic and nuclear localization. Heterologous overexpression of GhCYP-3 in Arabidopsis significantly improved Verticillium wilt resistance of the plants. Recombinant GhCYP-3 and the extracts from GhCYP-3 transgenic Arabidopsis displayed significantly inhibit activity to $V$. dahliae. These results indicated that GhCYP-3 was associated with the resistance of cotton to $V$. dahliae infection presumably through antifungal activity, and it will offer an important candidate gene for Verticillium wilt tolerance in cotton molecular breeding.

\section{Methods}

Identification and characterization of CYP family genes in G. hirsutum

Genome assemblies of G. hirsutum TM-1 from Nanjing Agricultural University (NAU version 1.1 and version 2.1 ) and JGI (version 1.0) were retrieved from the CottonFGD website (https://cottonfgd.org/). Arabidopsis CYPs, accessed from TAIR website (https://www.Arabidopsis. org/) were used as query to identify putative CYPs in G. hirsutum genomes by local BLAST using BioEdit software (Ibis Biosciences, Carlsbad, CA, USA). The identified GhCYPs were further verified through the Pfam database (http://pfam.xfam.org). The molecular weight (MW) and isoelectric point ( $\mathrm{pI}$ ) of each protein were calculated using ExPASy program (http://www.expasy.org/). The signal peptide was predicted with the program of SignalP 4.1. The amino acid sequences were aligned with DNAMAN software (Vers. 7; Lynnon Corporation, Quebec, Canada), using default parameters. CELLO v2.5 (http://cello.life.nctu.edu. tw/) was used for the subcellular localization prediction of GhCYPs. The putative cis-acting elements in the promoter regions were predicted using NAU version 2.1 database with the Plant CARE (http://bioinformatics.psb.ugent.be/ webtools/plantcare/html/).

\section{Genome-wide expression analysis of GhCYPs}

A genome-wide expression analysis of the cotton CYP genes in various abiotic stresses and $V$. dahliae infection was performed using high-through RNA sequence data, which was downloaded from NCBI databases (SRP044705) 
and extracted from our RNA-seq data [42]. Genes with FPKM $\geq 10$ were used for further expression analysis.

\section{Plant materials and $V$. dahliae strains}

The cotton seeds G. hirsutum cv. Ji Mian 20 (JM20), Han208, CCRI8 and G. barbadense cv. Pima90-53 were preserved at the North China Key Laboratory for Crop Germplasm Resources of Education Ministry, Hebei Agricultural University, Baoding, China. Cotton seedlings were grown in commercial sterilized soil at $28^{\circ} \mathrm{C}$ $125^{\circ} \mathrm{C}$ (day/night) temperatures with a 16 -h-light/8-hdark regime. A. thaliana was grown in pots containing vermiculite soil with temperature at $23^{\circ} \mathrm{C}$ day and $20^{\circ} \mathrm{C}$ night, under a $16 / 8 \mathrm{~h}$ photoperiod. $V$. dahliae strain Linxi2-1 was isolated from a symptomatic upland cotton plants growing in agricultural fields in Linxi county, Hebei Province, China [43]. These highly aggressive defoliating $V$. dahliae strains were maintained on PDA. The conidial suspension was prepared according to previous description [44] and adjusted to $10^{7}$ spores per milliliter and $10^{6}$ spores per milliliter with distilled water was used to the inoculation of cotton and Arabidopsis, respectively. The plant was infected with $V$. dahliae using soil drench method [44]. $10 \mathrm{~mL}$ of the conidial suspension was directly injected with a needle without piercing into the bottom of each pot. Seedlings received sterile water in the same manner were used as control.

\section{Gene cloning and subcellular localization}

Total RNA was extracted from leaf tissues of JM20 with an RNA plant plus reagent (TIANGEN Biotech, China). First-strand cDNA was synthesized from an aliquot of 1 $\mu \mathrm{g}$ of total RNA with a PrimeScript ${ }^{\text {tix }}$ RT Reagent Kit and gDNA Eraser (TaKaRa, China). GhCYP-3 was amplified with primers CYP-F1 and CYP-R1 (Additional file 1: Table S1), designed based on the sequences of Gh_A01G1361 (G. hirsutum L. acc. TM-1) [20]. A GhCYP-3-GFP fusion construct under the control of the $35 \mathrm{~S}$ promoter was generated by cloning the ORF into the SalI and BamHI sites of the binary vector pCamE. The vector expressing GFP alone served as control. Protein subcellular localization in onion (Allium cepa) epidermal cells was determined according to the protocol of Yang (2015) [44].

\section{qRT-PCR and semi-quantitative PCR}

Total RNA and cDNA were prepared by the method described above. The qRT-PCR was performed on a CFX96 Real-Time PCR Detection System (Bio-Rad, Hercules, CA, USA) using the qPCR kit for SYBR Green (TaKaRa, China). qPCR conditions consisted of one cycle of $3 \mathrm{~min}$ at $95{ }^{\circ} \mathrm{C}$, followed by 40 cycles of $15 \mathrm{~s}$ at $95{ }^{\circ} \mathrm{C}, 15 \mathrm{~s}$ at $58{ }^{\circ} \mathrm{C}$, and $20 \mathrm{~s}$ at $72{ }^{\circ} \mathrm{C}$. The cotton ubiquitin 14 (UBQ14) gene served as an internal standard [45]. Fold-changes in expression were calculated via the
$2^{-\Delta C t}$ method. Semi-quantitative RT-PCR was done with an Applied Biosystems ${ }^{\circ} 2720$ Thermal Cycler with Arabidopsis AtACT2 (AT3G18780) as the internal standard [46]. The resulting products were resolved on a $1.5 \%$ agarose gel. All primers are listed in Additional file 1: Table S1 Three biological and three technical replicates were analyzed for all quantitative experiments.

\section{Generation and evaluation resistance of transgenic Arabidopsis}

GhCYP-3 was cloned into pBI121 vector at the $X b a \mathrm{I}$ and $S a c$ I sites by PCR with primers CYP-X and CYP-S (Additional file 1: Table S1). The chimeric construct was introduced into Agrobacterium tumefaciens strain GV3101 for Arabidopsis transformation using the floral dip method [47]. Putative transformants were selected on MS (Murashige and Skoog) medium containing $50 \mathrm{mg}$ $\mathrm{L}^{-1}$ kanamycin, and then be further verified by PCR for gene insertion and semi-quantitative RT-PCR for gene expression. Independent $T_{1}$ transgenic lines were used to produce the $T_{3}$ generations, which were randomly chosen as representative lines and subjected to analysis. Disease severity of Arabidopsis plants caused by $V$. dahliae was assessed according to symptoms manifested on the leaves. The disease index (DI) was calculated as previously described [48]. Fungal recovery assay was also used to evaluate the resistance of the plant through assessment of Verticillium colonization recovered from stem sections according to the method of Fradin (2009) [49]. In each treatment, 18 individual plants were used and all the experiments were repeated thrice.

\section{Purification and PPlase activity assay of recombinant protein}

The GhCYP-3 ORF was cloned into expression vector pET-32a (+) (Novagen, Darmstadt, Germany) with forward primer CYP-Bg-F and reverse primer CYP-Sa-R (Additional file 1: Table S1), which will introduce the BglII and SacI site into the $5^{\prime}$ and $3^{\prime}$ end of the ORF, respectively. To induce the expression of GhCYP-3 protein with a His-tag in E. coli BL21(DE3) (TransGen Biotech, Beijing, China), a final concentration of $1.0 \mathrm{mmol} \cdot \mathrm{L}^{-1}$ isopropyl- $\beta$-D-thiogalactopyranoside (IPTG) was added into the culture when the $\mathrm{OD}_{600}$ value reached 0.4-0.6, and the culture was allowed to continue growing for $4-$ $6 \mathrm{~h}$ before harvesting. The proteins were separated on a SDS-PAGE gel and detected by western blot using anti His-Tag mouse monoclonal antibody (1:5000; CW Biotech, Beijing, China). The recombinant protein was purified using a $6 \times$ His-Tagged Protein Purification Kit (CW Biotech, Beijing, China). The PPIase activity of the recombinant protein was assayed in vitro using the tetrapeptide substrate Suc-AAPF-pNA (N-succinyl-Ala-LeuPro-Phe-p-nitroanilide; Sigma-Aldrich, Ontario, Canada) 
in a Shimadzu UV-2450 spectrophotometer (Shimadzu, Kyoto, Japan) as described by Yoon (2016) [12]. Three biological and two technical replicates were analyzed for all measurement.

\section{Assay of antifungal activity}

The antifungal activity of the recombinant protein was tested against $V$. dahliae using filter paper disc diffusion method. $V$. dahliae spores were uniformly spread on the PDA medium plates and then cultured for $60 \mathrm{~h}$ at $28^{\circ} \mathrm{C}$. Sterilized blank paper discs of $6 \mathrm{~mm}$ diameters, impregnated with tested protein, were placed on the surface of PDA medium previously spread with $V$. dahliae. The plates were inoculated at $25^{\circ} \mathrm{C}$ for $10 \mathrm{~d}$. The antifungal activity of extracts from Arabidopsis plants transformed with GhCYP-3 was performed as described previously [50] with the following modifications. Conidial suspension adjusted to a density of $10^{5}$ conidia $\mathrm{ml}^{-1}$, and germinated on PDA overnight at $25^{\circ} \mathrm{C}$ prior to assay. Total homogenates $(5 \mathrm{~g})$ from ten Arabidopsis plants were prepared by directly grinding plant leaves into a fine powder in liquid nitrogen with no buffer added. Subsequently, extracts were collected by centrifugation at $10000 \mathrm{~g}$ for $10 \mathrm{~min}$ at $25^{\circ} \mathrm{C}$. Conidial suspensions $(25 \mu \mathrm{l})$ were mixed with $225 \mu \mathrm{l}$ of extract, and incubated for $1 \mathrm{~h}$ at $25^{\circ} \mathrm{C}$. The mixture $(50 \mu \mathrm{l})$ were spread onto PDA plates and incubated at $25{ }^{\circ} \mathrm{C}$ for $48 \mathrm{~h}$ and fungal colonies enumerated. All experiments were repeated three times independently.

\section{Statistical analysis}

All experiments were performed at least three times for each determination. Statistical analysis was performed using GraphPad Prism 6 software (Graph Pad, San Diego, CA, USA). Unless otherwise indicated, data were evaluated by using analysis of variance (ANOVA) followed by Dunnett's multiple comparisons test.

\section{Additional files}

Additional file 1: Table S1. Primers list (DOCX $15 \mathrm{~kb}$ )

Additional file 2: Table S2. The transcripts of GhCYPS with FPKM in response to abiotic stress (XLSX $22 \mathrm{~kb}$ )

Additional file 3: Table S3. Putative CYPs identified in $V$. dahliae strain VdLs.17 (DOCX $13 \mathrm{~kb})$

\section{Abbreviations}

ABA: Abscisic acid; ABRE: Abscisic acid responsive element; CLD: Cyclophilin type PPlase domain; CYP: Cyclophilin; DI: Disease index; EDS1: Enhanced disease susceptibility 1; ERE: Ethylene responsive element; ET: Ethylene; FC: Fold change; FPKM: Fragments per kilobase of exon per million fragments mapped; GA: Gibberellin; hct: Hours after cold treated; hht: Hours after hot treated; hpi: Hours post inoculation; hpt: Hours after PEG treated; MeJA: Methyl jasmonate; MS: Murashige and Skoog; PAD4: Peptidyl arginine deiminase type 4; PDA: Potato dextrose agar; PEG: Polyethylene glycol: pl: Isoelectric point; PPlase: Peptidyl prolyl cis/trans isomerase; ROS: Reactive oxygen species; RRM: RNA recognition motif; SA: Salicylic acid;

TPR: Tetratricopeptide-like repeats; WT: Wild type

\section{Acknowledgments}

All authors are grateful to the laboratory members for help, advice and discussion.

\section{Authors' contributions}

$J Y, X W$ and ZM designed the experiments. JY carried out the study and wrote the manuscript. GW and LJ performed the qRT-PCR and evaluated the resistance of transgenic Arabidopsis to $\mathrm{V}$. dahliae. LH and CZ purified the recombinant protein and measured its antifungal activity. HK and YZ carried out the data analysis. All the authors have read and approved the publication of the manuscript.

\section{Funding}

This work was supported by grants from the National Key Research and Development Program of China (2016YFD0101006), the Top Talent Fund of Hebei Province and the Fund of Research Group Construction for Crop Science in Hebei Agricultural University (TD2016C328). None of the funding bodies were involved with the research other than providing funds to support the work.

\section{Availability of data and materials}

The data generated or analyzed during the current study are included in this published article and its supplemental data files and available from the corresponding author on reasonable request.

Ethics approval and consent to participate

Not applicable.

\section{Consent for publication}

Not applicable.

\section{Competing interests}

The authors declare that they have no competing interests.

Received: 2 January 2019 Accepted: 24 May 2019

Published online: 21 June 2019

\section{References}

1. Chen ZJ, Scheffler BE, Dennis E, Triplett BA, Zhang T, Guo W, Chen X, Stelly DM, Rabinowicz PD, Town CD, et al. Toward sequencing cotton (Gossypium) genomes. Plant Physiol. 2007;145(4):1303-10.

2. Zhang J, Flynn R, Baral JB, Bajaj S, Hughs SE, Percy RGJE. Germplasm evaluation and transfer of Verticillium wilt resistance from Pima (Gossypium barbadense) to Upland cotton (G. hirsutum). Euphytica. 2012;187(2):147-60.

3. Romano P, Gray J, Horton P, Luan S. Plant immunophilins: functional versatility beyond protein maturation. New Phytol. 2005;166(3):753-69.

4. Olga B, Marek Z, Yamile M, Tatsiana S, Maria K, Andrea BJNAR. Identification of RNA targets for the nuclear multidomain cyclophilin atCyp59 and their effect on PPlase activity. Nucleic Acids Res. 2013:41(3):1783-96.

5. Kumari S, Roy S, Singh P, Singla-Pareek SL, Pareek A. Cyclophilins: proteins in search of function. Plant Signaling \& Behavior. 2013;8(1):e22734.

6. Kristine NB, Mallis RJ, Fulton DB, Andreotti AH. Regulation of the tyrosine kinase Itk by the peptidyl-prolyl isomerase cyclophilin A. Proc Natl Acad Sci U S A. 2002:99(4):1899-904

7. Hong F, Lee J, Song JW, Lee SJ, Ahn H, Cho JJ, Ha J, Kim SS. Cyclosporin A blocks muscle differentiation by inducing oxidative stress and inhibiting the peptidyl-prolyl-cis-trans isomerase activity of cyclophilin A: cyclophilin A protects myoblasts from cyclosporin Ainduced cytotoxicity. FASEB J. 2002;16(16):1633-5.

8. Romano PG, Horton P, Gray JE. The Arabidopsis cyclophilin gene family. Plant Physiol. 2004;134(4):1268-82.

9. Juncheul A, Daewon K, You YN, Minsook S, Jeongmee P, Hyunsik H, Beomgi K, Sheng L, Hongseog P, Hyesun C. Classification of rice (Oryza sativa L. japonica Nipponbare) immunophilins (FKBPs, CYPs) and expression patterns under water stress. BMC Plant Biol. 2010;10(1):1-22.

10. Mainali HR, Chapman P, Dhaubhadel S. Genome-wide analysis of cyclophilin gene family in soybean ( Glycine max ). BMC Plant Biol. 2014;14(1):1-11.

11. Chou IT, Gasser CS. Characterization of the cyclophilin gene family of Arabidopsis thaliana and phylogenetic analysis of known cyclophilin proteins. Plant Mol Biol. 1997;35(6):873. 
12. Yoon DH, Sang SL, Park HJ, Lyu Jl, Chong WS, Liu JR, Kim BG, Ahn JC, Cho HS. Overexpression of OsCYP19-4 increases tolerance to cold stress and enhances grain yield in rice (Oryza sativa). J Exp Bot. 2016;67(1):69-82.

13. Lee SS, Park HJ, Jung WY, Lee A, Yoon DH, You YN, Kim HS, Kim BG, Ahn JC, Cho HS. OsCYP21-4, a novel Golgi-resident cyclophilin, increases oxidative stress tolerance in rice. Front Plant Sci. 2015;6:797.

14. Sekhar K, Priyanka B, Reddy VD, Rao KV. Isolation and characterization of a pigeonpea cyclophilin (CCCYP) gene, and its over-expression in Arabidopsis confers multiple abiotic stress tolerance. Plant Cell Environ. 2010;33(8):1324-38.

15. Kong HY, Lee SC, Hwang BK. Expression of pepper cyclophilin gene is differentially regulated during the pathogen infection and abiotic stress conditions. Physiol Mol Plant Pathol. 2001;59(4):189-99.

16. Godoy AV, Lazzaro AS, Casalongué CA, San Segundo B. Expression of a Solanum tuberosum cyclophilin gene is regulated by fungal infection and abiotic stress conditions. Plant Sci. 2000;152(2):123-34.

17. Figueiredo A, Monteiro F, Pais MS, Rex M, Töpfer R, Zyprian E. Cyclophilin: A downy mildew resistance gene candidate in grapevine. Acta Horticulturae. 2014;1046(1046):371-8.

18. Zhu C, Wang Y, Li Y, Bhatti KH, Tian Y, Wu J. Overexpression of a cotton cyclophilin gene (GhCyp 1) in transgenic tobacco plants confers dual tolerance to salt stress and Pseudomonas syringae pv. tabaci infection. Plant Physiol Biochem. 2011;49(11):1264-71.

19. Li FG, Fan GY, Lu CR, Xiao GH, Zou CS, Kohel RJ, Ma ZY, Shang HH, Ma $X F$, Wu JY, et al. Genome sequence of cultivated Upland cotton (Gossypium hirsutum TM-1) provides insights into genome evolution. Nat Biotechnol. 2015;33(5):524-30.

20. Zhang TZ, Hu Y, Jiang WK, Fang L, Guan XY, Chen JD, Zhang JB, Saski CA, Scheffler BE, Stelly DM, et al. Sequencing of allotetraploid cotton (Gossypium hirsutum L. acc. TM-1) provides a resource for fiber improvement. Nat Biotechnol. 2015:33(5):531-7.

21. Hu Y, Chen J, Fang L, Zhang Z, Ma W, Niu Y, Ju L, Deng J, Zhao T, Lian J, et al. Gossypium barbadense and Gossypium hirsutum genomes provide insights into the origin and evolution of allotetraploid cotton. Nat Genet. 2019;51(4):739-48.

22. Trivedi DK, Yadav S, Vaid N, Tuteja N. Genome wide analysis of cyclophilin gene family from rice and Arabidopsis and its comparison with yeast. Plant Signal Behav. 2012;7(12):1653.

23. Chen Q, Chen Q-J, Sun G-Q, Zheng K, Yao Z-P, Han Y-H, Wang L-P, Duan YJ, Yu D-Q, Qu Y-Y. Genome-wide identification of cyclophilin gene family in cotton and expression analysis of the fibre development in Gossypium barbadense. Int J Mol Sci. 2019;20(2):349.

24. Albà MM, Pagès M. Plant proteins containing the RNA-recognition motif. Trends Plant Sci. 1998;3(97):15-21.

25. Smith TF, Gaitatzes C, Saxena K, Neer EJ. The WD repeat: a common architecture for diverse functions. Trends Biochem Sci. 1999;24(5):181-5.

26. Sane AP, Stein B, Westhoff P. The nuclear gene HCF107 encodes a membrane-associated R-TPR (RNA tetratricopeptide repeat)-containing protein involved in expression of the plastidial psbH gene in Arabidopsis. Plant J. 2005:42(5):720-30.

27. Chen L, Hamada S, Fujiwara M, Zhu T, Thao NP, Wong HL, Krishna P, Ueda T, Kaku H, Shibuya N, et al. The Hop/Sti1-Hsp90 chaperone complex facilitates the maturation and transport of a PAMP receptor in rice innate immunity. Cell Host Microbe. 2010;7(3):185-96.

28. Dirk W, Roman B, Joachim S. The regulatory code for transcriptional response diversity and its relation to genome structural properties in $A$. thaliana. Plos Genetics. 2007;3(2):e11.

29. Wang C, Liu Y, Li S-S, Han G-Z. Insights into the origin and evolution of plant hormone signaling machinery. Plant Physiol. 2015;167(3):872-86.

30. Zhang CY, Wang XF, Zhang GY, Lq W, Chi JN, Li ZK, Ma ZY. ESTs analysis of suppression subtractive hybridization library from upland cotton resistant cultivar infected by Verticillium dahliae. Cotton Sci. 2010;22(01):17-22.

31. Saito T, Niwa Y, Ashida H, Tanaka K, Kawamukai M, Matsuda H, Nakagawa T. Expression of a gene for cyclophilin which contains an amino-terminal endoplasmic reticulum-targeting signal. Plant Cell Physiol. 1999:40(1):77-87.

32. Pogorelko GV, Mokryakova M, Fursova OV, Abdeeva I, Piruzian ES, Bruskin SA. Characterization of three Arabidopsis thaliana immunophilin genes involved in the plant defense response against Pseudomonas syringae. Gene. 2014;538(1):12-22.

33. Mainali HR, Vadivel AK, Li X, Gijzen M, Dhaubhadel S. Soybean cyclophilin GmCYP1 interacts with an isoflavonoid regulator GmMYB176. Sci Rep. 2017;7:39550.
34. Kong G, Zhao Y, Jing M, Huang J, Yang J, Xia Y, Kong L, Ye W, Xiong Q, Qiao Y. The activation of phytophthora effector Avr3b by plant cyclophilin is required for the nudix hydrolase activity of Avr3b. PLoS Pathog. 2015;11(8):e1005139.

35. Zhang H, Wang J, Li S, Wang S, Liu M, Wang W, Zhao Y. Molecular cloning, expression, purification and functional characterization of an antifungal cyclophilin protein from Panax ginseng. Biomedical Reports. 2017;7(6):527-31.

36. Lee JR, Park S-C, Kim J-Y, Lee SS, Park Y, Cheong G-W, Hahm K-S, Lee SY. Molecular and functional characterization of a cyclophilin with antifungal activity from Chinese cabbage. Biochem Biophys Res Commun. 2007;353(3):672-8.

37. Ye XY, Ng TB. Isolation of a new cyclophilin-like protein from chickpeas with mitogenic, antifungal and anti-HIV-1 reverse transcriptase activities. Life Sci. 2002;70(10):1129-38

38. Ye XY, Ng TB. Isolation of Unguilin, a cyclophilin-like protein with antimitogenic, antiviral, and antifungal activities, from Black-Eyed Pea. J Protein Chem. 2001:20(5):353-9.

39. Theis T, Stahl U. Antifungal proteins: targets, mechanisms and prospective applications. Cell Mol Life Sci. 2004;61(4):437-55.

40. Chan BP, Kim HS, Sun CK. Mechanism of action of the antimicrobial peptide Buforin II: Buforin II kills microorganisms by penetrating the cell membrane and inhibiting cellular functions. Biochem Biophys Res Commun. 1998;244(1):253-7.

41. Zhou TT, Zhao YL, Guo HS. Secretory proteins are delivered to the septinorganized penetration interface during root infection by Verticillium dahliae. PLoS Pathog. 2017:13(3):e1006275.

42. Zhang Y, Wang X, Rong W, Yang J, Li Z, Wu L, Zhang G, Ma Z. Histochemical analyses reveal that stronger intrinsic defenses in Gossypium barbadense than in G. hirsutum are associated with resistance to Verticillium dahliae. Mol Plant Microbe Interact. 2017;30(12):984-96.

43. Wang GN, Zhao GY, Yue XW, Li ZK, Zhang Y, Zhang GY, Wu LQ, Wang $X F$, Ma ZY. Pathogenicity and ISSR genetic differentiation of Verticillium dahliae isolates from cotton growing areas of Hebei Province. Cotton Science. 2012;24(4):348-57.

44. Yang J, Ji L, Wang X, Zhang Y, Wu L, Yang Y, Ma Z. Overexpression of 3-deoxy7-phosphoheptulonate synthase gene from Gossypium hirsutum enhances Arabidopsis resistance to Verticillium wilt. Plant Cell Rep. 2015;34(8):1429-41.

45. Artico S, Nardeli SM, Brilhante O, Grossi-de-Sa MF, Alves-Ferreira M. Identification and evaluation of new reference genes in Gossypium hirsutum for accurate normalization of real-time quantitative RT-PCR data. BMC Plant Biol. 2010;10:49.

46. Czechowski T, Stitt M, Altmann T, Udvardi MK, Scheible W-R. Genome-wide identification and testing of superior reference genes for transcript normalization in Arabidopsis. Plant Physiol. 2005;139(1):5-17.

47. Clough SJ, Bent AF. Floral dip: a simplified method for Agrobacteriummediated transformation of Arabidopsis thaliana. Plant J. 1998;16(6):735-43.

48. Zhang B, Yang Y, Chen T, Yu W, Liu T, Li H, Fan X, Ren Y, Shen D, Liu L, et al. Island cotton Gbvel gene encoding a receptor-like protein confers resistance to both defoliating and non-defoliating isolates of Verticillium dahliae. PLoS One. 2012:7(12):e51091.

49. Fradin EF, Zhang Z, Juarez Ayala JC, Castroverde CD, Nazar RN, Robb J, Liu CM, Thomma BP. Genetic dissection of Verticillium wilt resistance mediated by tomato Ve1. Plant Physiol. 2009;150(1):320-32.

50. Cary JW, Jaynes JM, Cleveland TRK. Transgenic expression of a gene encoding a synthetic antimicrobial peptide results in inhibition of fungal growth in vitro and in planta. Plant Sci. 2000;154(2):171-81.

\section{Publisher's Note}

Springer Nature remains neutral with regard to jurisdictional claims in published maps and institutional affiliations. 